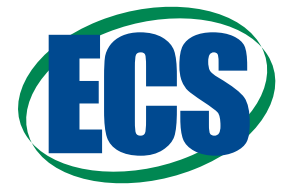

JeS Focus Issue on Mathematical Modeling of Electrochemical Systems at Multiple Scales in Honor of John Newman

\title{
Revealing SEI Morphology: In-Depth Analysis of a Modeling Approach
}

\author{
Fabian Single, ${ }^{\mathrm{a}, \mathrm{b}, \mathrm{z}}$ Birger Horstmann,,${ }^{\mathrm{a}, \mathrm{b}, \mathrm{z}}$ and Arnulf Latz ${ }^{\mathrm{a}, \mathrm{b}, \mathrm{c}}$ \\ ${ }^{a}$ German Aerospace Center (DLR), Institute of Engineering Thermodynamics, 70569 Stuttgart, Germany \\ ${ }^{b}$ Helmholtz Institute Ulm (HIU), 89081 Ulm, Germany \\ ${ }^{c}$ Ulm University, Institute of Electrochemistry, 89069 Ulm, Germany
}

\begin{abstract}
In this article, we present a novel theory for the long term evolution of the solid electrolyte interphase (SEI) in lithium-ion batteries and propose novel validation measurements. Both SEI thickness and morphology are predicted by our model as we take into account two transport mechanisms, i.e., solvent diffusion in the SEI pores and charge transport in the solid SEI phase. We show that a porous SEI is created due to the interplay of these transport mechanisms. Different dual layer SEIs emerge from different electrolyte decomposition reactions. We reveal the behavior of such dual layer structures and discuss its dependence on system parameters. Model analysis enables us to interpret SEI thickness fluctuations and link them to the rate-limiting transport mechanism. Our results are general and independent of specific modeling choices, e.g., for charge transport and reduction reactions.

(C) The Author(s) 2017. Published by ECS. This is an open access article distributed under the terms of the Creative Commons Attribution 4.0 License (CC BY, http://creativecommons.org/licenses/by/4.0/), which permits unrestricted reuse of the work in any medium, provided the original work is properly cited. [DOI: 10.1149/2.0121711jes] All rights reserved.

(cc) BY
\end{abstract}

Manuscript submitted February 6, 2017; revised manuscript received April 17, 2017. Published May 5, 2017. This paper is part of the JES Focus Issue on Mathematical Modeling of Electrochemical Systems at Multiple Scales in Honor of John Newman.

In the near future, automotive and mobile applications demand power storage with large energy and power density. Currently, lithiumion batteries (LIBs) are the technology of choice for devices with these demands. They operate at high cell potentials and offer high specific capacities while providing long lifetimes. The latter is a consequence of the stable chemistry of modern LIB systems. A significant part of this stability can be attributed to the passivation ability of the solid electrolyte interphase (SEI). This thin layer forms between the negative electrode and the electrolyte. Hence contact between these phases is prevented and the continuous reduction of electrolyte molecules is suppressed. These reduction processes occur because the operating potential of the negative electrode lies well below the stability window of the electrolyte. ${ }^{1}$ They are suppressed because reduction products quickly form the SEI during the first charge of a pristine electrode. The self passivating ability is one of the most important distinctions between a well and a badly performing lithium-ion battery chemistry. It is of such importance because the reduction reactions consume lithium-ions, directly reducing battery capacity. However, a real SEI is not perfectly passivating and electrolyte reduction is never completely suppressed. Consequently, the lifetime of a battery is directly related to the long-term passivating ability of the SEI.

Numerous studies on SEI have been conducted since Peled reported on this correlation in $1979 .^{2}$ Most of these studies are experimental, investigating cycling stability as well as SEI impedance and composition. Theoretical studies are scarce in comparison, despite established methods such as DFT and DFT/MD derivatives. This can be partially explained with the chemical diversity of SEI, which has been investigated by Aurbach et al. for decades. Results are summarized in Refs. 3, 4 and include the study of SEI formation on graphite electrodes in organic solvent mixtures. The most significant finding of this time is that ethylene carbonate (EC) forms a stable SEI on graphite as opposed to propylene carbonate (PC). Another focus of early studies is the SEI composition, which has been probed by FTIR and XPS and other techniques. Lithium carbonate $\left(\mathrm{Li}_{2} \mathrm{CO}_{3}\right)$ and lithium alkyl carbonates have been reported as products from the reduction of organic carbonates.

Studies of simplified systems, i.e., binder-free electrodes have improved our understanding of SEI composition only recently. ${ }^{5}$ This advance is also due to the use of novel experimental techniques such as solid state NMR and TEM. ${ }^{6,7}$ The focus of these studies are the standard $\mathrm{LiPF}_{6} /$ organic carbonate mixtures on graphite and silicon anodes.
They find that SEI in EC containing solvents is primarily composed of lithium ethylene dicarbonate $\left(\left(\mathrm{CH}_{2} \mathrm{OCO}_{2} \mathrm{Li}\right)_{2}, \mathrm{Li}_{2} \mathrm{EDC}\right)$. Polyethylene oxide is also found as a major product of $\mathrm{EC}$ reduction. Linear carbonates like dimethyl carbonate (DMC) are reduced to lithium alkyl carbonates, such as lithium methyl carbonate $\left(\mathrm{CH}_{3} \mathrm{OCO}_{2} \mathrm{Li}, \mathrm{LiMC}\right)$. These compounds play a secondary role when EC is in the solvent mixture. This is linked to the solvation shell of lithium-ions which are preferably coordinated to EC. ${ }^{6,8}$ Furthermore, EC has a higher reduction potential. ${ }^{9} \mathrm{Li}_{2} \mathrm{CO}_{3}$ is not present or only found in small quantities in recent studies. ${ }^{6,7,10}$ Its presence in several older studies is believed to correlate to water and $\mathrm{CO}_{2}$ contamination.

The electrolyte salt has a large impact on SEI composition and performance. It can shift the onset potential of SEI formation and influence the total irreversible capacity during the first cycle. ${ }^{10,11} \mathrm{In}$ $\mathrm{LiPF}_{6}$ solutions, $\mathrm{LiF}$ is another major SEI compound while lithium oxyflurophosphates $\left(\mathrm{Li}_{x} \mathrm{PF}_{y} \mathrm{O}_{z}\right)$ are present in low quantities. ${ }^{12}$ The complex $\mathrm{LiPF}_{6}$ decomposition process is investigated by Campion and Lux. ${ }^{13,14}$

Additionally, SEI composition depends on the electrode material. Solvent decomposition reactions proceed differently on graphite and lithium storage alloys. ${ }^{15}$ Electrode materials exhibiting large volume change, i.e., silicon, fail to form a stable SEI. SEI needs to be flexible to accommodate volume changes of the underlying substrate without damage by cracking or rupture. It is believed that these properties can be, to some degree, provided by polymeric SEI compounds as found when FEC is used as solvent or additive. ${ }^{12}$ Harris and $\mathrm{Lu}^{16,17}$ show, that SEI consists of a porous outer layer and a dense inner (close the the electrode) layer by using isotope tracer and depth profiling techniques such as TOF-SIMS. Evidence for a dual-layer structure is found in the chemical composition of the film. Solid state NMR studies also suggest that SEI is at least partially porous.

To summarize, there is a general understanding of SEI composition and morphology for few specific systems. Especially SEIs on graphite electrodes in organic solvents are studied and optimized for battery performance in several studies. This vast amount of information creates the elusive conclusion that SEI is well understood. However, several key questions about basic SEI mechanisms have yet to be answered. Most striking is the fact that the mechanism for lithium-ion transport through the SEI is still debated. Shi et al. propose a "knock-of" diffusion mechanism for lithium-ion interstitials in $\mathrm{Li}_{2} \mathrm{CO}_{3} \cdot{ }^{18}$ Diffusion of lithium-ions through $\mathrm{Li}_{2} \mathrm{EDC}$ is modeled by Borodin et al. ${ }^{19}$ At the same time Zhang et al. suggest that lithium-ions diffuse and migrate along boundaries between different SEI species. ${ }^{20}$ Another open question is the process of initial SEI formation where 
nucleation and precipitation could play an important role. Ushirogata et al. have recently suggested a "near-shore aggregation" mechanism of electrolyte decomposition products. ${ }^{21}$ This is supported by the fact that the occupation of the lithium-ion solvation shell seems to have a large impact on SEI properties, ${ }^{6,8}$ which suggests that reduction reactions occur in solution. Alternatively, solvent molecules could be reduced when adsorbed to the electrode. In this case, reduction products could attach to the electrode immediately. Finally, there is an open discussion about the mechanism driving long term SEI growth. The passivation of the SEI is not perfect and irreversible reduction reactions continue throughout the battery life..$^{22,23}$ This could be enabled by several different mechanisms, for example electron leakage through the SEI. However, a porous SEI allowing slow solvent diffusion through the film is equally plausible. In this scenario, solvent molecules would reach the electrode if the SEI is porous or ruptured by the "breathing" of the underlying electrode.

The lack of information on these issues can be attributed to several reasons. The results of many common experimental techniques are to some degree ambiguous. Interpretations of FTIR and XPS spectra are difficult because many SEI compounds are similar to each other and to residual electrolyte within the sample. ${ }^{24}$ Rinsing the sample of excess electrolyte before the measurement is common, but known to selectively damage SEI. Therefore, SEI is difficult to access experimentally. Furthermore, too many variables influence SEI properties significantly, preventing a systematic investigation. Not only the solvent/salt combination but also the electrode material and its surface treatment influence SEI formation and properties. ${ }^{25}$ Formation can take place at different potentials, cycling rates and temperatures. Finally, SEI chemistry is known to be sensitive to air exposure which often occurs during sample transfer. All this makes analyzing and comparing different studies and their results difficult. Especially the identification of universal SEI properties and mechanisms becomes complicated.

Continuum theories describe SEI formation in a simplified way and elucidate such universal properties. In this way, they circumvent specifying the reaction kinetics of the SEI formation reaction. Instead, the formation rate is limited and determined by the throughput of the so called "rate-limiting" transport mechanism. These models assume one such mechanism as the cause for long-term SEI growth, i.e., electron conduction ${ }^{26,27}$ and tunneling ${ }^{28,29}$ or solvent/salt diffusion. ${ }^{30,31}$ However, independent of the assumed mechanism, all of these models predict similar long-term SEI thickness evolution. Therefore, even a successful measurement of this prediction cannot be used to confirm the underlying assumptions.

In conclusion, theoretical models based on transport through the SEI should predict additional measurable properties and dependencies. Tang et al. ${ }^{32}$ approach this task by comparing experiments with different models, each based on a single rate-limiting mechanism. Because of the dependence of SEI growth rate on electrode potential, they finally conclude that electron conduction rather than solvent diffusion is rate-limiting.

For this reason, we extend the standard approach, using two potentially rate-limiting transport mechanisms at the same time and modeling a dynamic SEI porosity. This is done in a one dimensional framework. We describe the evolution of SEI thickness and morphology along the axes perpendicular to the electrode surface. The overall objective of this work is to make new observable predictions which allow to test and validate our assumptions. Besides thickness evolution, our model can predict the formation of a porous SEI and explain several dual-layer scenarios. These results are obtained for two different rate-limiting transport mechanisms namely electron conduction and diffusion of neutral lithium interstitials. Additionally, solvent diffusion through the SEI pores can become the rate-limiting transport mechanism. In fact we can smoothly transition the rate-limiting role from the solid phase transport mechanism to solvent diffusion. This reveals an intermediate regime where both transport mechanisms influence the formation rate.

The model and its numerical implementation are discussed in the Model and Model implementation section. We then proceed to study

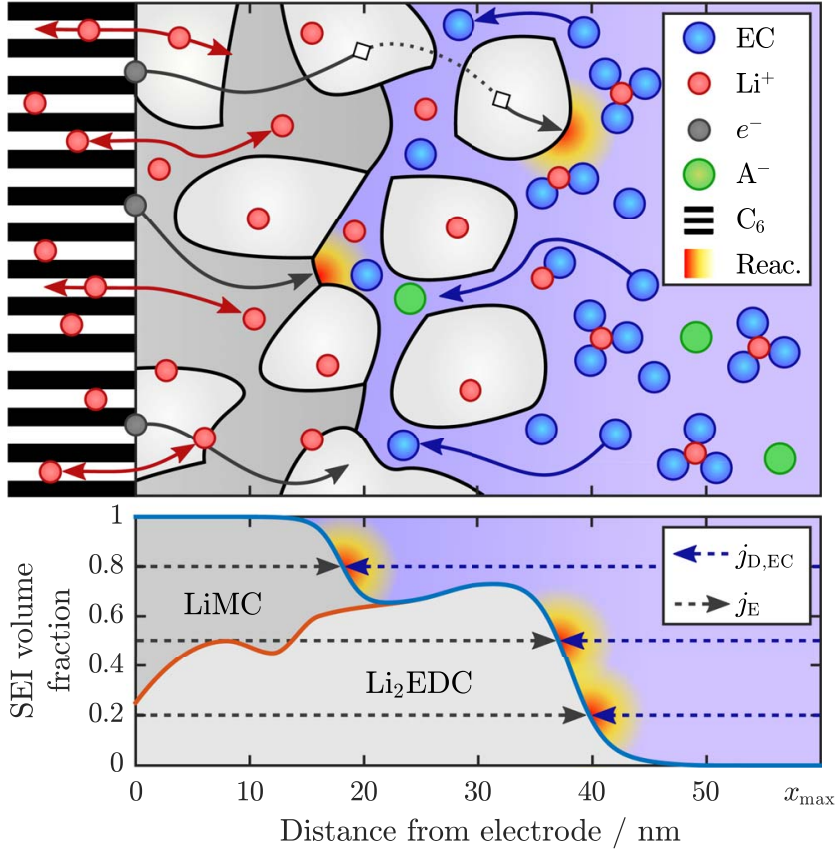

Figure 1. (a) Cross section through the graphite electrode (left, $x<0$ ), and a SEI with dual layer structure (right, $x>0$ ). Solvent, Li-ions and electrons are mobile species and move as indicated by the corresponding arrows. Reduction reactions (indicated red), consume these species and facilitate SEI growth. (b) SEI volume fraction gained by averaging the structure above in planes parallel to the electrode surface.

different sets of model scenarios, in the Simulation results section. In this way, we show how measurable SEI properties depend on specific assumptions and allow their experimental verification. First, we study our reference scenario, a SEI formed by a single reduction reaction. Then, the impact of an additional SEI formation reaction is studied. This slightly more complex SEI chemistry results in the observed duallayer structure of the SEI. At the end of the results section we evaluate the effect of material laws dictating a minimum value of the SEI porosity. We find that solvent diffusion can become the rate-limiting transport mechanism under this assumption. We conclude the Simulation results section by illustrating for which parameter set solvent diffusion in the electrolyte or charge transport in the SEI are ratelimiting. We elaborate how these mechanisms can be distinguished by experiments. Finally, we discuss and summarize our results in two dedicated sections.

\section{Model}

As mentioned above, experimental studies suggest that the SEI is at least partially porous. We want to capture this property in our model. Therefore, we average the SEI density in planes parallel to the electrode surface. This results in the volume fraction profile of the SEI $\varepsilon_{\text {SEI }}$, as depicted in Fig. 1. Our model describes the temporal evolution of this profile within the simulation domain $\left[0, x_{\max }\right]$ which reaches from the electrode surface at $x=0$ into the bulk electrolyte phase. We capture the local formation of each individual SEI compound $i=\mathrm{Li}_{2} \mathrm{EDC}, \mathrm{LiMC}, \ldots$ which changes the corresponding volume fraction $\varepsilon_{i}$

$$
\frac{\partial \varepsilon_{i}}{\partial t}=\bar{V}_{\mathrm{SEI}}^{i} \dot{n}_{i}
$$

where $\dot{n}_{i}$ is the production rate of SEI compound $i$ and $\bar{V}_{\text {SEI }}^{i}$ is the corresponding partial molar volume. The total SEI volume fraction equals the sum of solid phase volume fractions $\varepsilon_{i}$. It is directly related 
to porosity $\varepsilon$

$$
\varepsilon_{\mathrm{SEI}}=\sum_{i} \varepsilon_{i}, \quad \varepsilon_{\mathrm{SEI}}=1-\varepsilon .
$$

SEI is formed when solvent or salt species are reduced. Reduction processes are driven by local quantities such as the electronic potential and the concentration of active species. These quantities are traced within the simulation domain as they determine the reduction rates. Therefore, mass balance equations are solved for all relevant electrolyte species

$$
\frac{\partial \varepsilon c_{i}}{\partial t}=-\operatorname{div}\left(j_{\mathrm{M}, i}+j_{\mathrm{D}, i}+j_{\mathrm{C}, i}\right)+\dot{n}_{i},
$$

where div denotes the divergence, $\operatorname{div} j=\nabla \cdot j$. Migration of charged species $\left(j_{\mathrm{M}, i}\right)$ and diffusion $\left(j_{\mathrm{D}, i}\right)$ are the microscopic processes which transport particles inside the electrolyte. Together with convection $\left(j_{\mathrm{C}, i}\right)$ they determine the evolution of $c_{i}$, the concentration of electrolyte species $i=\mathrm{EC}$, DMC. A source term $\dot{n}_{i}$ couples the concentrations to consumption by reduction reactions, see Eq. 11 . The local porosity $\varepsilon$ appears on the left-hand side as we use porous electrode theory to describe the mass balance within the nano-porous SEI. ${ }^{33}$

As mentioned in the introduction, SEI chemistry is complex and highly dependent on the lithium-ion battery chemistry. Our framework is capable of modeling this chemistry in detail for each system individually, however such a realization requires many parameters which are not readily available. Large amounts of parameters for transport and reaction kinetics would make the identification of qualitatively significant results difficult. We simplify SEI chemistry and consider only one or two representative reduction reactions.

Reduction reactions take place at the interface between solid SEI material and the liquid electrolyte. SEI products precipitate immediately. Furthermore, the influence of charged species within the electrolyte is simplified. We assume that the electrochemical potential of lithium-ions is in equilibrium and constant. Lithium consumption due to SEI growth does not perturb the concentration locally because $\mathrm{Li}^{+}$mobility inside the SEI is very high compared to the rate of SEI formation. Furthermore the salt anion is neglected as an active species.

To summarize, solvent molecules are the only electrolyte species considered in our simulation. Assuming a binary mixture of solvent and co-solvent, two mass balance equations of type Eq. 2 are solved. Fickian diffusion and convection are the relevant transport processes for these species

$$
j_{\mathrm{D}, i}=-D_{i} \nabla c_{i}, \quad j_{\mathrm{C}, i}=c_{i} v, \quad j_{\mathrm{M}, i}=0,
$$

where $D_{i}$ is the effective diffusion coefficient and $v$ the convection velocity in the center of mass frame. One mass balance equation can be eliminated with the constitutive relation ${ }^{34}$

$$
1=\sum_{i} \bar{V}_{\text {Elyte }}^{i} c_{i}, \quad \text { yielding } 0=\sum_{i} \bar{V}_{\text {Elyte }}^{i} \nabla c_{i}
$$

Here, we assume that partial molar volumes $\bar{V}_{\text {Elyte }}^{i}$ are constant and independent of concentration. By summing all mass balance equations (see Eq. 2) multiplied with $\bar{V}_{\text {Elyte }}^{i}$, we obtain

$\operatorname{div} v=\operatorname{div} \sum_{i} \bar{V}_{\text {Elyte }}^{i} D_{i} \nabla c_{i}-\frac{\partial \varepsilon}{\partial t}=\bar{V}_{\text {Elyte }}^{\mathrm{EC}} \operatorname{div}\left(D_{\mathrm{EC}}-D_{\mathrm{DMC}}\right) \nabla c_{\mathrm{EC}}-\frac{\partial \varepsilon}{\partial t}$

In the second line, we applied Eq. 4 to a binary solvent mixture of EC and DMC specifically. This definition of the convection velocity ensures that all pores are filled with an incompressible electrolyte. ${ }^{35,36}$ Because $v$ is the center of mass velocity, the diffusive mass fluxes in the electrolyte add up to zero

$$
\sum_{i} M_{i} j_{\mathrm{D}, i}=0
$$

where $M_{i}$ is the molar mass of solvent species $i$. Thus, in the binary mixture, both diffusion coefficients are related, $M_{\mathrm{EC}} D_{\mathrm{EC}} \bar{V}_{\text {Elyte }}^{\mathrm{DMC}}=$ $M_{\mathrm{DMC}} D_{\mathrm{DMC}} \bar{V}_{\text {Elyte }}^{\mathrm{EC}}$.
In the solid SEI phase, we take a second transport mechanism into account. This mechanism needs to transport a reduced species or electrons from the electrode/SEI interface through the SEI. As discussed in the Simulation results section, our results do not depend on the specific transport mechanism chosen. This is important because several different mechanisms seem plausible. For simplicity, we use electron conduction inside the solid SEI phase in our reference case. According to Ohm's law, the electronic current is driven by a potential gradient

$$
j_{\mathrm{E}}=-\kappa \nabla \Phi,
$$

where $\kappa$ is the effective electronic conductivity, assumed equal for all SEI compounds. $j_{\mathrm{E}}$ is an electron leakage current through the SEI which fuels SEI formation and is much smaller than the lithium-ion intercalation current. Charge conservation is modeled by coupling the current to the reaction rate of each individual reaction

$$
0=-\operatorname{div} j_{\mathrm{E}}+F \sum_{j} n^{j} r_{j}
$$

Here, $n^{j} r_{j}$ is the rate of electron consumption of reduction reaction $j$.

We consider faradaic surface reactions. Each reaction $j$ is of the general type

$$
\sum_{i} \tilde{s}_{i}^{j} \mathrm{~S}_{i}+n^{j}\left(\mathrm{Li}^{+}+e^{-}\right) \rightarrow \sum_{k} s_{k}^{j} \mathrm{~S}_{k},
$$

where $\tilde{s}_{i}^{j}$ and and $s_{i}^{j}$ are the stoichiometric coefficients. The sums include all electrolyte species and SEI compounds. In our simplified SEI chemistry each solvent reacts to a single SEI compound. Therefore, we use the solvent precursor as the reaction index $j=\mathrm{EC}$, DMC. Reaction rates $r_{j}$, see Eq. 8, are determined with symmetric Butler-Volmer expressions, see recent works by Latz and Bazant, ${ }^{37,38}$ or standard literature, e.g., 33,39

$$
r_{j}=\frac{k_{\mathrm{B}} T}{h} \exp \left(\frac{-E_{\mathrm{A}}}{k_{\mathrm{B}} T}\right) \prod_{i}\left(\frac{c_{i}}{c_{i}^{0}}\right)^{\frac{\tilde{s}_{i}}{2}} \sinh \frac{n^{j} F \eta_{j}}{R T},
$$

where $E_{\mathrm{A}}$ is the energy difference between the initial and the transition state.

The overpotential $\eta_{j}$ is the driving force of reaction $j$ and will be discussed below. Oxidation of SEI compounds is only possible at high voltages ( $>3.25 \mathrm{~V}$, see ref. 40$)$ which are not met in normal battery operation. Generally, anodic reactions do not occur in our simulations because we always polarize the electrode below the onset potential of SEI formation. However, we need to actively prevent anodic reactions if a second SEI compound is considered. This is achieved by using $\tilde{\eta}_{j}=\max \left(0, \eta_{j}\right)$ for these reactions.

Source terms $\dot{n}_{i}$ in Eqs. 1 and 2, consist of the sum over all reduction reactions

$$
\dot{n}_{i}=\sum_{j}\left(s_{i}^{j}-\tilde{s}_{i}^{j}\right) \rho_{j} r_{j},
$$

where $\rho_{j}$ is the reaction site density which depends on the type of the reaction $j$. It equals $\varepsilon_{j} / \bar{V}_{\text {SEI }}^{j}$ for bulk reactions in the solid SEI phase. For solvent reduction reactions which occur at the interface between solid SEI material and the liquid electrolyte, $\rho_{j}$ equals the product $\Gamma A$, where $A$ is the specific surface area and $\Gamma$ is the surface site density. $A$ is a function of porosity, as discussed below, while $\Gamma$ is assumed constant.

SEI formation reactions.-As mentioned above, every reaction considered in our model introduces additional parameters. Therefore, we simplify SEI chemistry. We study all reactions listed below in different combinations, namely I, I + II and I + III. This means we consider up to two reactions at a time.

We assume a single reduction reaction for solvent and co-solvent

$$
2 \mathrm{EC}+2 \cdot\left(\mathrm{Li}^{+}+e^{-}\right) \rightarrow \mathrm{Li}_{2} \mathrm{EDC}+R,
$$




$$
\mathrm{DMC}+\mathrm{Li}^{+}+e^{-} \rightarrow \mathrm{LiMC}+R .
$$

Gaseous by-products $R$ are neglected in our simulation, as they quickly escape the simulation domain. Given the potential $\Phi$ and the concentration of each electrolyte species, we can express the overpotential for these reactions.

$$
\begin{array}{r}
\eta_{\mathrm{EC}}=\Phi_{\mathrm{EC}}^{0}-\Phi+\frac{1}{2} \frac{R T}{F} \ln \left(\frac{c_{\mathrm{EC}}}{c_{\mathrm{EC}}^{0}}\right), \\
\eta_{\mathrm{DMC}}=\Phi_{\mathrm{DMC}}^{0}-\Phi+\frac{1}{4} \frac{R T}{F} \ln \left(\frac{c_{\mathrm{DMC}}}{c_{\mathrm{DMC}}^{0}}\right),
\end{array}
$$

where $\Phi_{i}^{0}$ is the reduction onset potential of solvent species $i$ and $c_{i}^{0}$ is the corresponding reference concentration.

It is known that SEI species are to some degree unstable, especially at low potentials. ${ }^{41}$ Therefore, aside from solvent molecules, SEI compounds can be reduced as well, forming new compounds and by-products. Lithium oxide $\left(\mathrm{Li}_{2} \mathrm{O}\right)$ has been reported as SEI compound which is mostly found close to the electrode surface. ${ }^{17,42}$ Therefore, we assume the formation of $\mathrm{Li}_{2} \mathrm{O}$ by successive reduction of $\mathrm{Li}_{2} \mathrm{EDC}^{41}$

$$
0.1 \mathrm{Li}_{2} \mathrm{EDC}+\mathrm{Li}^{+}+e^{-} \rightarrow 0.6 \mathrm{Li}_{2} \mathrm{O}+0.4 \mathrm{C},
$$

where $\mathrm{C}$ denotes carbon. We have normalized this reaction to one lithium-ion, i.e., electron respectively. We calculate the kinetics of this reaction with Eq. 10. The overpotential of conversion reactions has no concentration contribution

$$
\eta_{\mathrm{Li}_{2} \mathrm{EDC}}=\Phi_{\mathrm{Li}_{2} \mathrm{EDC}}^{0}-\Phi .
$$

Solid convection.- - Independent of the specific conversion reaction chosen, a volume mismatch between the educts and products is typical. This volume mismatch creates an "excess volume" when the reaction is ongoing. Excess volume can be accommodated by a porosity change or by a displacement of the whole SEI such that porosity remains constant at the location of the reaction. We consider a mixture of both mechanisms by adding solid convection to the model and defining a suitable solid convection velocity $\tilde{v}$. Convective fluxes need to be considered in Eq. 1, which is therefore modified

$$
\frac{\partial \varepsilon_{i}}{\partial t}=\bar{V}_{\mathrm{SEI}}^{i} \dot{n}_{i}-\operatorname{div} \varepsilon_{i} \tilde{v} .
$$

In two extreme cases, the solid convection velocity is given as

$$
\begin{gathered}
\varepsilon_{\mathrm{SEI}} \operatorname{div} \tilde{v}=0, \\
\varepsilon_{\mathrm{SEI}} \operatorname{div} \tilde{v}=\sum_{j=\mathrm{conv}} \Delta \bar{V}_{\mathrm{SEI}}^{j} \rho_{j} r_{j},
\end{gathered}
$$

where the sum includes all conversion reactions. $\Delta \bar{V}_{\text {SEI }}^{j}$ is the excess molar volume of the reaction. When the porosity is high, volume changes of individual SEI particles do not induce solid convection, as established by Eq. 15a. In Eq. 15b, the convection velocity is defined such that SEI porosity remains unchanged locally. Therefore, the SEI expands in response to SEI formation. Such a behavior can be expected when the porosity is almost zero and SEI cannot become any denser.

We model a smooth transition from local accumulation to SEI expansion as the SEI becomes denser. The solid convection velocity is calculated from

$$
\varepsilon_{\mathrm{SEI}} \operatorname{div} \tilde{v}=\alpha\left(\varepsilon_{\mathrm{SEI}}\right) \sum_{j=\text { conv }} \Delta \bar{V}_{\mathrm{SEI}}^{j} \rho_{j} r_{j},
$$
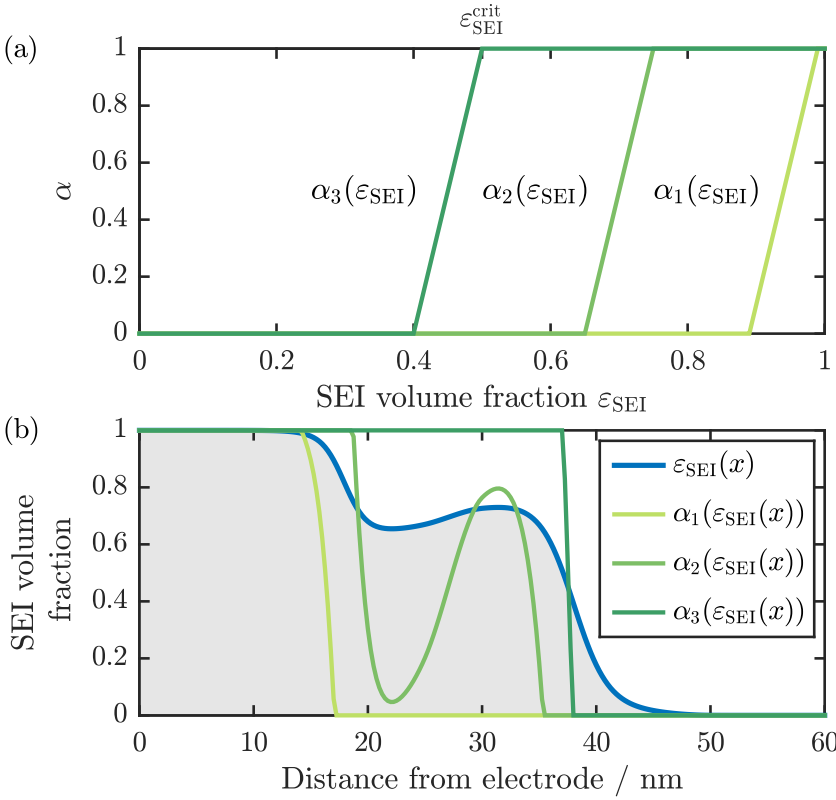

Figure 2. (a) $\alpha\left(\varepsilon_{\mathrm{SEI}}\right)$ as a function of the SEI volume fraction for $\varepsilon_{\mathrm{SEI}}^{\mathrm{crit}}=$ $0.99,0.75$ and 0.5 , see Eq. 17. The location of the critical value is indicated for $\alpha_{3}\left(\varepsilon_{\text {SEI }}\right)$. (b) Spatial dependence of $\alpha$ for a given SEI volume fraction $\varepsilon_{\text {SEI }}$.

where $\alpha\left(\varepsilon_{\text {SEI }}\right)$ models a smooth transition between Eqs. $15 \mathrm{a}$ and $15 \mathrm{~b}$. This transition is performed in a linear way

$$
\alpha\left(\varepsilon_{\mathrm{SEI}}\right)= \begin{cases}0 & \varepsilon_{\mathrm{SEI}} \leq \varepsilon_{\mathrm{SEI}}^{\text {crit }}-\Delta \alpha, \\ 1 & \varepsilon_{\mathrm{SEI}} \geq \varepsilon_{\mathrm{SEI}}^{\mathrm{crit}}, \\ 1+\frac{\varepsilon_{\mathrm{SEI}}-\varepsilon_{\mathrm{SEI}}^{\text {crit }}}{\Delta \alpha} & \text { otherwise. }\end{cases}
$$

Here $\Delta \alpha$ is the width of the transition, chosen to be 0.1 . The influence of the empirical parameter $\varepsilon_{\mathrm{SEI}}^{\text {crit }}$ on SEI formation will be studied in the Simulation results section. It is one unless mentioned otherwise. It constitutes the greatest volume fraction that the SEI material can reach. Several versions of $\alpha\left(\varepsilon_{\mathrm{SEI}}\right)$, differing in the choice of this parameter are shown in Fig. 2a.

Transport in porous media.-The local porosity $\varepsilon$ determines the phase distribution in our simulation domain. Pure electrolyte and SEI phase are represented by $\varepsilon=1$ and $\varepsilon=0$, respectively. If $\varepsilon$ is between zero and one, both electrolyte and SEI phase are present, arranged in a porous structure. As each transport mechanism is restricted to a single phase only, the corresponding transport parameters have to depend on the porosity. They decrease with the volume fraction of the phase they belong to. We use the Bruggeman ansatz to describe this behavior, i.e., we use a power law to relate these parameters at a given porosity to their bulk values. Bruggeman coefficients encode the structural information of the porous structure which is lost when averaging to obtain a one dimensional system. High values of $\beta$ correspond to large tortuosity. The effective diffusion coefficient depends on the porosity

$$
D_{i}=\varepsilon^{\beta} D_{i}^{\text {Bulk }},
$$

where the Bruggeman coefficient $\beta$ is a parameter in our model whose influence will be part of our study. Analogously, electron conduction scales with the SEI volume fraction

$$
\kappa=\varepsilon_{\text {SEI }}^{1.5} \kappa^{\text {Bulk }} .
$$

We have chosen 1.5 as the Bruggeman coefficient for transport in the solid SEI phase because it is the standard value. Percolation effects are not considered by this description. Therefore transport through a phase remains possible until the phase disappears completely, i.e., if $\varepsilon=0$ or $\varepsilon_{\mathrm{SEI}}=0$ 
Specific surface area.-Solvent reduction and SEI formation take place at the interface between solid SEI material and the liquid electrolyte. Consequently, the source term of solvent reduction reactions is directly proportional to the specific surface area $A$ (see Eq. 11). The specific surface area depends on the local porosity. We derive an approximation for this dependence from the assumption that SEI particles and pores are arranged on a cubic lattice with edge length $a_{0}$. This parameter corresponds to the average particle and pore size of the SEI. We consider a large volume $V \gg a_{0}^{3}$ in which all sub-cubes are randomly assigned to SEI/pores with uniform probability $\varepsilon_{\mathrm{SEI}} / \varepsilon$. The total surface area in $V$ can be approximated as

$$
A_{\text {total }} \approx \frac{V}{a_{0}^{3}} \cdot 6 \cdot a_{0}^{2} \cdot \varepsilon \varepsilon_{\mathrm{SEI}},
$$

where $\mathrm{Va}_{0}^{-3}$ is the number of cubes. Every cube has six surfaces, each with an area of $a_{0}^{2}$. The probability of a cube being empty while a neighboring cube is filled equals the product $\varepsilon \varepsilon_{\text {SEI }}$. Here, surfaces on the edge of $V$ have been neglected. Then the specific surface area of $V$ reads

$$
A=A_{\text {total }} / V=\frac{6}{a_{0}} \varepsilon \varepsilon_{\mathrm{SEI}} .
$$

We need to adjust this expression because we study porosity profiles, this means porosity changes in one direction. To this aim, we study a slice $V$ with the thickness of a single cube $a_{0}$. Now, surfaces on the edge of $V$ can no longer be neglected and have to be taken into account. Therefore, we use the SEI volume fraction of the neighboring slices

$$
A=\frac{\varepsilon}{a_{0}}\left(4 \varepsilon_{\mathrm{SEI}}+\varepsilon_{\mathrm{SEI}}\left(x-a_{0}\right)+\varepsilon_{\mathrm{SEI}}\left(x+a_{0}\right)\right) .
$$

Using a second order Taylor expansion for $\varepsilon_{\mathrm{SEI}}\left(x \pm a_{0}\right)$ we find

$$
A(\varepsilon) \approx \frac{6}{a_{0}} \varepsilon\left(\varepsilon_{\mathrm{SEI}}+\frac{a_{0}^{2}}{6} \frac{\partial^{2} \varepsilon_{\mathrm{SEI}}}{\partial x^{2}}\right) .
$$

In comparison to Eq. 21, an effective, non-local SEI volume fraction replaces the local value. This modification enables growth into the pure electrolyte phase where $\varepsilon_{\mathrm{SEI}}$, and thus $A$ according to Eq. 21, is zero.

This approximation is good, when the porosity changes slowly in space relative to $a_{0}$, i.e., $\left|\partial_{x}^{2} \varepsilon\right|<2 a_{0}^{-2}$. If $\varepsilon(x)$ has a larger curvature, the Taylor expansion is not valid and Eq. 22 can become negative. However, these situations are averted in our simulations and the small quantitative errors do not influence our main results.

Regularization.-During our simulation SEI is formed and $\varepsilon_{\mathrm{SEI}}$ increases. When $\varepsilon_{\text {SEI }}$ reaches unity at a certain location, a pure SEI phase would be formed. Pure phases are numerically difficult because transport equations for the absent phase become ambiguous. To avoid such problems, we implement two regularizations.

We prevent the formation of a dense SEI with vanishing porosity. This is achieved by modifying the specific surface area such that $\varepsilon<1-\Delta \varepsilon$ is guaranteed at all times

$$
\tilde{A}\left(\varepsilon, \varepsilon_{\mathrm{SEI}}^{\prime \prime}\right)=\frac{6}{a_{0}}(\varepsilon-\Delta \varepsilon)\left(\varepsilon_{\mathrm{SEI}}+\frac{a_{0}^{2}}{6} \frac{\partial^{2} \varepsilon_{\mathrm{SEI}}}{\partial x^{2}}\right),
$$

where $\Delta \varepsilon=0.001$ is small. Mass balance equations, see Eq. 2, are guaranteed to be well defined with this modification.

In a pure electrolyte phase, equation Eq. 8 cannot be used to solve for the potential as $\kappa=\varepsilon_{\text {SEI }}^{1.5} \kappa^{\text {Bulk }}$ is zero. This can be alleviated by using

$$
\kappa=\left[\varepsilon_{\mathrm{SEI}}^{1.5}+\Delta \exp \left(-\varepsilon_{\mathrm{SEI}}^{2} / \Delta\right)\right] \kappa^{\text {Bulk }},
$$

which is equal to the Bruggeman relation at small porosities and attains $\Delta \cdot \kappa^{\text {Bulk }}$ as $\varepsilon \rightarrow 1$. This numerical procedure is necessary because our classical continuum theory cannot describe microscopic quantum effects. We describe here the spatial extend of the reaction process as the microscopic cause for SEI expansion. Therefore, the small conductivity in the electrolyte enables SEI growth into the electrolyte phase. We choose $\Delta=0.05$, quite large compared to $\Delta \varepsilon$. Hence, charge transport into the electrolyte phase is a negligible barrier and does not affect our simulation results. At the same time, we make sure that the electron current does not reach beyond a few $\AA$ into the electrolyte.

\section{Model Implementation}

Initialization and boundary conditions.-We begin our simulations at $t=0$. Initially the system is in a stationary state, which means that all reactions are in equilibrium. Consequently, the initial potential and concentration are chosen such that all overpotentials are zero, i.e., $\Phi(x, 0)=\Phi_{\mathrm{EC}}^{0}$ and $c_{\mathrm{EC}}(x, 0)=c_{\mathrm{EC}}^{0}$. Thus, both convection velocities vanish, $v=\tilde{v}=0$. The volume fraction of $\mathrm{Li}_{2} \mathrm{EDC}$ is zero apart from a small region next to the electrode $\varepsilon_{\mathrm{Li}_{2} \mathrm{EDC}}(x>2 \mathrm{~nm}, 0)=0$. An initialization profile serves as nucleation seed

$\frac{\varepsilon_{\mathrm{Li}_{2} \mathrm{EDC}}(x<2 \mathrm{~nm}, 0)}{1-\Delta \varepsilon}=-\frac{3}{16}\left(\frac{x}{\mathrm{~nm}}\right)^{5}+\frac{15}{16}\left(\frac{x}{\mathrm{~nm}}\right)^{4}-\frac{5}{4}\left(\frac{x}{\mathrm{~nm}}\right)^{3}+1$.

where $x$ is the distance from the electrode. The volume fraction changes smoothly from $1-\Delta \varepsilon \approx 1$ to zero, as shown in Fig. 4a. It represents the roughness of the electrode surface and adsorption layers of SEI formed at voltages above $0.8 \mathrm{~V}$. The thickness correlates to the critical thickness SEI can reach by electron tunneling, as predicted by Lin et al. ${ }^{29}$ The volume fraction of the second SEI compound considered is zero initially.

The simulation domain spans from the electrode surface at $x=$ 0 into the bulk electrolyte at $x=x_{\max }$. We choose our boundary conditions such that they describe the contact to these phases. While the electrode is a "reservoir" for the electronic current it acts as an impenetrable boundary for the electrolyte. Therefore diffusive and convective fluxes vanish at this interface. Solvent can be drawn from the right-hand side boundary at which electronic currents must vanish.

$$
\begin{aligned}
\Phi(0, t) & =\Phi_{\mathrm{OCV}}(t), \quad j_{\mathrm{E}}\left(x_{\max }, t\right)=0, \\
j_{\mathrm{D}, \mathrm{EC}}(0, t) & =0, \quad c_{\mathrm{EC}}\left(x_{\max }, t\right)=c_{\mathrm{EC}}^{0}, \\
v(0, t) & =0, \quad \tilde{v}(0, t)=0,
\end{aligned}
$$

where $\Phi_{\mathrm{OCV}}(t)$ is determined from the state of charge (SOC) of a graphite electrode taken from Ref. 43. SOC is ramped linearly such that the electrode potential $\Phi(t)$ decreases from $\Phi_{\mathrm{EC}}^{0}$ at $t=0$ to the final electrode potential $\Phi_{\mathrm{E}}$ in 20 hours. Then SOC and potential remain constant, representing storage conditions. We stop the simulations shortly before SEI growth reaches $x_{\max }$. In this way we make sure that the right boundary does not influence the results.

Parameterization.-All parameters used, for example, to create the data for figures and the results discussed, are summarized in Table I. They are listed in four groups according to their type.

- The molar volume of each SEI species determines the evolution rate of the corresponding SEI volume fraction, see Eq. 1. The molar volumes of electrolyte species define the amplitude of convection velocities induced by volume mismatch during reduction reactions in Eq. 5 and Eq. 16.

- Bulk diffusivity and conductivity in solvent and SEI are needed to calculate the electron and solvent flux. The Bruggeman coefficient is used to calculate the effective diffusion coefficient in the nano-porous SEI, see Eq. 18.

- Reaction rates are determined by a couple of parameters, e.g. the transition energy $E_{\mathrm{A}}$ and the pore size of the SEI structure $a_{0}$. The latter determines the area available for reactions, see Eq. 22.

- The equilibrium of each reaction is characterized by an equilibrium potential and a reference concentration, see Eq. 12. 
Table I. List of simulation parameters, all potentials relative to the $\mathrm{Li} / \mathrm{Li}^{+}$reduction pair.

\begin{tabular}{|c|c|c|}
\hline Parameter & Description & Value Unit \\
\hline $\bar{V}_{\text {Elyte }}^{\text {EC }}$ & EC molar volume & $66.7 \mathrm{~cm}^{3} / \mathrm{mol}^{44}$ \\
\hline $\bar{V}_{\text {Elyte }}^{\text {DMC }}$ & DMC molar volume & $84.2 \mathrm{~cm}^{3} / \mathrm{mol}^{44}$ \\
\hline $\bar{V}_{\mathrm{SEI}}^{\mathrm{Li}_{2} \mathrm{EDC}}$ & $\mathrm{Li}_{2} \mathrm{EDC}$ molar volume & $96.2 \mathrm{~cm}^{3} / \mathrm{mol}^{45}$ \\
\hline $\bar{V}_{\mathrm{SEI}}^{\mathrm{LiMC}}$ & LiMC molar volume & $58.1 \mathrm{~cm}^{3} / \mathrm{mol}^{45}$ \\
\hline $\bar{V}_{\mathrm{SEI}}^{\mathrm{LIEC}}$ & LiEC molar volume & $58.1 \mathrm{~cm}^{3} / \mathrm{mol}$ \\
\hline $\bar{V}_{\mathrm{SEI}}^{\mathrm{Li} \mathrm{i}_{2} \mathrm{O}}$ & $\mathrm{Li}_{2} \mathrm{O}$ molar volume & $14.9 \mathrm{~cm}^{3} / \mathrm{mol}^{45}$ \\
\hline$\kappa^{\text {Bulk }}$ & Conductivity of all SEI compounds & $1 \mathrm{pS} / \mathrm{m}$ \\
\hline$D^{\text {Bulk }}$ & EC diffusion coefficient & $10^{-10} \mathrm{~cm}^{2} / \mathrm{s} 46$ \\
\hline$\beta$ & Bruggeman coefficient for solvent diffusion & $20-$ \\
\hline$\varepsilon_{\text {crit }}$ & Critical (lowest possible) SEI porosity & $0.8,0.9-$ \\
\hline$a_{0}$ & Pore-size and size of SEI particles & $1.0 \mathrm{~nm}$ \\
\hline$\Gamma$ & Surface site density & $4.0 \mu \mathrm{mol} / \mathrm{m}^{2} 45$ \\
\hline$E_{\mathrm{A}}$ & Transition state energy & $1.0 \mathrm{eV}$ \\
\hline$c_{\mathrm{EC}}^{0}$ & EC concentration in bulk electrolyte & $4.5 \mathrm{~mol} / 1$ \\
\hline$\Phi_{\mathrm{EC}}^{0}$ & EC reduction potential & $0.8 \mathrm{~V}^{16}$ \\
\hline$\Phi_{\mathrm{DMC}}^{0}$ & DMC reduction potential & $0.3 \mathrm{~V}^{16}$ \\
\hline$\Phi_{\mathrm{Li}_{2} \mathrm{EDC}}^{0}$ & DMC reduction potential & $0.3 \mathrm{~V}^{16}$ \\
\hline$\Phi_{\mathrm{E}}$ & Electrode potential during simulation & $0.1 \mathrm{~V}$ \\
\hline
\end{tabular}

We assume that LiEC has the same partial molar volume as LiMC due to the similarity between both molecules. The Bruggeman coefficient $\beta=20$ is chosen to describe the slow effective mesoscopic transport of solvent within the SEI nano-pores, whose microscopic mechanism is not understood. Furthermore, large values of $\beta$ lead to larger porosities and allow easier illustration, e.g., in Fig. 4a.

Note that the other relevant symbols are listed and described in Table AI.

Numerical implementation.-Numerical methods.-We solve equations $2,5,8,14$, and 16 on a static and equidistant grid spanning from 0 to $x_{\max }$. All equations are solved for the primary variables $\varepsilon_{i}, c_{\mathrm{EC}}, \Phi, v$, and $\tilde{v}$ in the whole domain at all times. The domain size in this work is $60 \mathrm{~nm}$. All equations are discretized with the finite volume method which ensures continuity of mass and charge. Convective currents, e.g. $j_{\mathrm{C}, \mathrm{EC}}=c_{\mathrm{EC}} v$ are calculated on the boundaries between discretization units. To calculate these currents we use the concentration of the left or right neighbor volume, depending on the sign of the velocity. This is done for solid convection as well. All simulations were performed in MATLAB with the implicit solver ODE15i.

SEI front properties.- In our simulations, we observe no SEI formation reactions inside the pores of the SEI or at the electrode/SEI interface.

Instead, reactions take place at the interphase separating the inner, homogeneous SEI from the pure electrolyte phase. This interphase has a width of roughly $1 \mathrm{~nm}$ and is called SEI front below. The porosity increases over the SEI front until it reaches unity, marking the end of the SEI and the beginning of the electrolyte phase, shown in Fig. 3. It is of vital importance that transport and reaction kinetics are solved with the necessary precision at the SEI front. We find that this is only possible, when the discretization is sufficiently fine, i.e., when a high resolution grid is used. The necessary resolution depends on the form of the front, which in turn depends on the small set of parameters and model assumptions

- activation energy $E_{\mathrm{A}}$, see Eq. 10 ,

- specific surface area $A\left(\varepsilon, \varepsilon^{\prime \prime}\right)$, see Eq. 22,
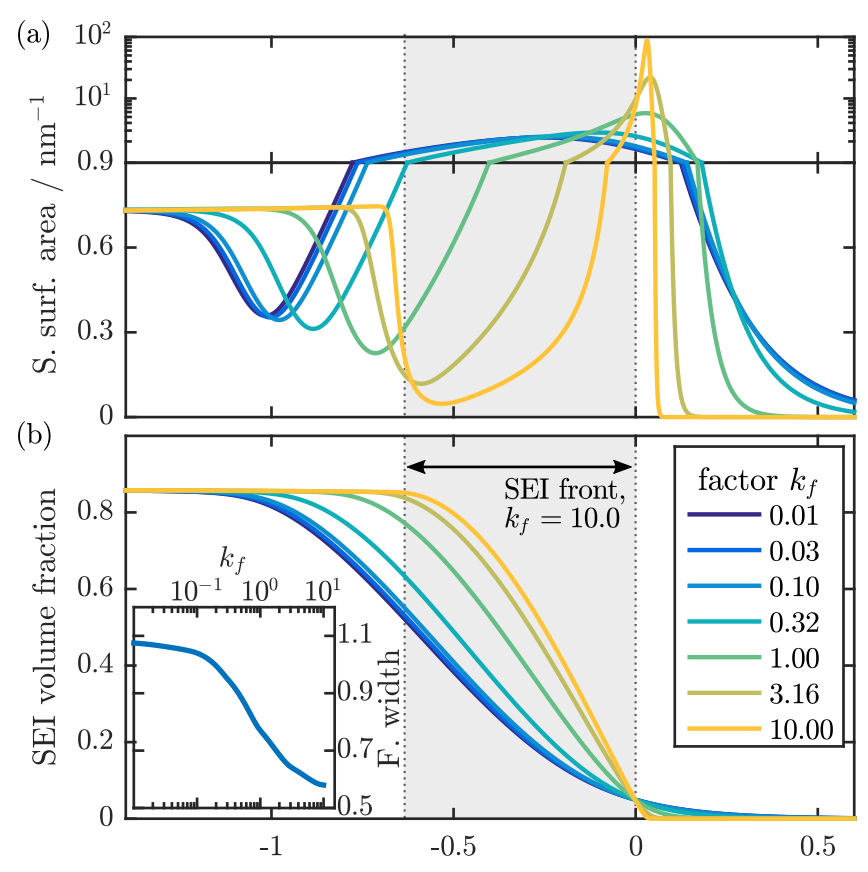

Distance from $x=L / \mathrm{nm}$

Figure 3. Specific surface area at the SEI front (a), see Eq. 22 (upper part scaled logarithmically) and the corresponding SEI volume fraction (b). Both plots show simulation results which differ only in the kinetic rate scale factor $k_{\mathrm{f}}$ in Eq. 26. The region we refer to as SEI front is marked gray for the simulation with $k_{f}=10.0$. The inset shows how the front width depends on $k_{\mathrm{f}}$.

These parameters influence the shape of the SEI front and the distribution of SEI formation within this region. They impact the thickness evolution and SEI porosity, two important results of our model. We therefore want to dedicate this subsection to discuss how the front shape is affected by these parameters. To do so, we have to go far afield.

Because of the boundary condition $j_{\mathrm{E}}\left(x_{\max }\right)=0$, all electronic charge transported through the SEI must be consumed at the front. Consequently, the overpotential $\eta$ will adjust itself such that electron consumption by SEI formation reactions at the front balances the incoming current $j_{\mathrm{E}}(x=0)$. In the following, we speak of slow reactions, when this overpotential is large $(\eta>10 \mathrm{mV})$. Reactions are fast, when the overpotential is small $(\eta<1 \mathrm{mV})$. Note that the total reaction turnover at the front is almost the same in both cases, only the necessary driving force is different.

To probe the difference between SEI formation in the slow and in the fast regime, we introduce the scale factor $k_{\mathrm{f}}$. This factor is only used in this section and modifies the reaction rate

$$
r_{j}=k_{\mathrm{f}} \frac{k_{\mathrm{B}} T}{h} \exp \left(\frac{-E_{\mathrm{A}}}{k_{\mathrm{B}} T}\right) \prod_{i}\left(\frac{c_{i}}{c_{i}^{0}}\right)^{\frac{\tilde{s}_{i}}{2}} \sinh \frac{n^{j} F \tilde{\eta}_{j}}{R T} .
$$

When we increase $k_{\mathrm{f}}$, the overpotentials decrease and reactions become fast. In return overpotentials become larger as we decrease $k_{\mathrm{f}}$ and we enter the slow regime.

As shown in Fig. 3b, the shape of the SEI front changes significantly with $k_{\mathrm{f}}$. When reactions are slow, the front is wide and smooth. It becomes thinner as the reaction rate increases. At the same time, the specific surface area changes with the front shape, see Fig. 3a. It becomes smaller in the region where the porosity increases. Furthermore, we observe the formation of a singularity which emerges if the front width $\Delta L$ is smaller than $a_{0}$. As explained in the Model section, our expression for $A$ (see Eq. 22) is derived for a slowly varying porosity. This is not the case when reactions are fast and the front width is below $a_{0}$ (see inset of Fig. 3b). To avoid this we adjusted our kinetics such that the width of the SEI front is wide $\left(\Delta L>a_{0}\right)$ during 
our simulation by choosing $E_{\mathrm{A}}$ accordingly. Therefore the scale factor $k_{\mathrm{f}}$ is not used below $\left(k_{\mathrm{f}}=1\right)$.

Whether reactions are fast or slow depends on the overpotential at the front. This overpotential does not remain constant during the evolution of the SEI. It decreases because the electronic current through the SEI decreases as the SEI becomes thicker, see Eq. 27. The reactions become slower due to the decreasing influx of electrons. Therefore, the SEI front becomes wider during SEI formation.

Numeric convergence.-We find that the grid resolution necessary to obtain well converged simulations depends on the shape of the SEI front. Any "kink" in this profile (such as visible in Fig. $3 \mathrm{~b}$ for $k_{\mathrm{f}}=10$ at $x \approx 0$ ), needs to be resolved sufficiently well. If not, the specific surface area will have an almost singular point because it is a function of the second spatial derivative of the porosity profile, see Eq. 22. Not only is this situation costly to solve numerically, it also influences the SEI porosity and growth rate by a few percent. However, we observe a directed and fast convergence of these quantities when the grid parameter becomes small enough. For example, when comparing two porosity profiles of the same simulation, performed with different grid parameters ( 2 and $0.66 \mathrm{pm}$ ), the largest difference inside the SEI is approximately $10^{-5}$.

\section{Simulation Results}

This section contains four subsections which address potential scenarios of SEI formation. We begin with our reference scenario, the formation of a chemically homogeneous SEI before discussing growth scenarios with higher complexity. Then, we study dual-layer SEIs obtained by adding a second SEI formation reaction. We proceed by taking mechanical properties of the SEI into account so that solvent diffusion can become rate-limiting. Finally, we discuss how the form of the rate limiting transport mechanism affects observable quantities. In this way, we increase the model complexity step-by step and systematically predict SEI properties based on specific sets of assumptions.

Single-layer SEI.-In this section, we study SEI formation assuming an inert co-solvent. This means that SEI formation is represented by a single reduction reaction, i.e., the reduction of $\mathrm{EC}$ to $\mathrm{Li}_{2} \mathrm{EDC}$, see Reaction I. We are able to derive analytic expressions for our primary results in this reference scenario. Our simulations start with a nearly uncharged graphite electrode which is charged to $\Phi_{\mathrm{E}}=0.1 \mathrm{~V}$ in the first 20 hours of the simulation. The electrode potential is then kept constant, simulating long-term storage under open-circuit condition. Fig. 4a shows the temporal evolution of the corresponding SEI volume fraction. We observe the formation of a porous film which gradually becomes thicker in our simulations. SEI formation occurs at the SEI front, shown in Fig. 3, indicating that electron conduction is the rate-limiting transport mechanism. No reactions take place inside the SEI where porosity remains constant in time. Both, the rate of SEI growth and the SEI porosity depend on the simulation parameters. We study this parameter dependence below, where we refer to the average porosity of a specific simulation as $\varepsilon^{*}=1-\varepsilon_{\mathrm{SEI}}^{*}$.

Thickness evolution.-The formation of SEI species is located at the front of the film and thus causes lateral growth. Therefore electron conduction is limiting the rate of SEI growth. This is reflected in the SEI potential which increases linearly from $\Phi_{\mathrm{E}}$ to $\Phi_{\mathrm{EC}}^{0}$ at any given time, shown in Fig. $4 \mathrm{~b}$. The electronic current $j_{\mathrm{E}}$ within the SEI is constant and electrons are transported from the electrode to the SEI front. We use this observation to approximate the electronic current $j_{\mathrm{E}}$ through the SEI

$$
\begin{aligned}
j_{\mathrm{E}}(x) & =-\kappa\left(\varepsilon_{\mathrm{SEI}}(x)\right) \nabla \Phi(x) \approx-\kappa^{*} \frac{\Phi(L)-\Phi(0)}{L}, \\
& \approx-\kappa^{*} \frac{\Phi_{\mathrm{EC}}^{0}-\Phi_{\mathrm{E}}}{L},
\end{aligned}
$$
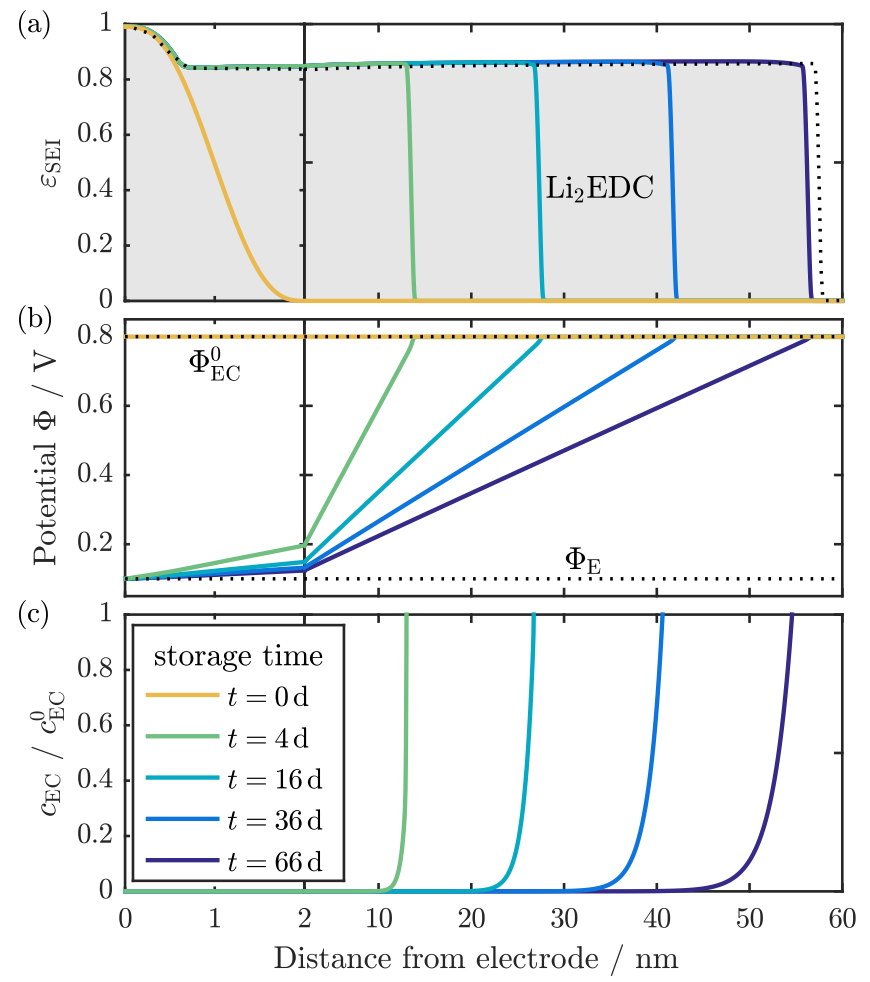

Figure 4. (a) SEI volume fraction $\varepsilon_{\mathrm{SEI}}(x, t)$ at various times of the simulation. Note the different scaling of the $\mathrm{x}$-axes to highlight the initial SEI profile. The dashed line is a profile from a simulation where neutral lithium interstitial diffusion has been used as the rate-limiting transport mechanism. (b) Potential $\Phi(x, t)$ and, (c) relative solvent concentration $c_{\mathrm{EC}}(x, t)\left(c_{\mathrm{EC}}^{0}=4.5 \mathrm{moll}^{-1}\right)$.

where $\kappa^{*}=\varepsilon_{\mathrm{SEI}}^{* 1.5} \kappa^{\text {Bulk }}$. We can couple this current directly to the evolution rate of the SEI thickness $L$,

$$
\frac{\partial L}{\partial t}=\frac{\bar{V}_{\mathrm{SEI}}^{\mathrm{Li}_{2} \mathrm{EDC}}}{2} \frac{1}{\varepsilon_{\mathrm{SEI}}^{*}} \frac{\left(-j_{\mathrm{E}}\right)}{F},
$$

where the first fraction takes the density of the SEI material and the stoichiometry of the formation reaction into account. The second fraction factors in film porosity. Finally, the Faraday constant $F$ converts the current density into a particle flux density.

Using approximation 27 in Eq. 28 results in a first order differential equation for $L$. The solution

$$
L(t)=\sqrt{\frac{\kappa^{*} \Delta \Phi_{\mathrm{EC}} \bar{V}_{\mathrm{SEI}}^{\mathrm{Li}_{2} \mathrm{EDC}}}{\varepsilon_{\mathrm{SEI}}^{*} F}} \sqrt{t},
$$

depends on the mean film porosity $\varepsilon^{*}$, which is not a parameter but a result of our simulation. The parameter dependence of this value is very complex and will be discussed later. Eq. 29 has the well known $\sqrt{t}$ dependence of transport limited growth. We observe this time dependence of SEI thickness in our simulations, see Fig. 5a. The expression agrees well with simulation results as shown on the right side of this figure. Small derivations can be linked to the offset between $\Phi(L)$ and $\Phi_{\mathrm{EC}}^{0}$. However this error is in the order of few $\mathrm{mV}$ and small compared to $\Delta \Phi_{\mathrm{EC}}=\Phi(L)-\Phi_{\mathrm{EC}}^{0} \approx 700 \mathrm{mV}$.

As seen in Eq. 29, only a few parameters influence the growth rate directly. These are the conductivity $\kappa^{\text {Bulk }}$, the molar volume of $\mathrm{Li}_{2} \mathrm{EDC}$ and the applied potential $\Phi_{\mathrm{E}}$. Other parameters, such as $\beta$ and $D^{\text {Bulk }}$ can influence the average SEI porosity $\varepsilon^{*}$, which in turn affects the thickness evolution. However, as shown in Fig. 5a, the influence of $\varepsilon^{*}$ on the growth rate is small. Consequently, assuming an inaccurate porosity in Eq. 29 only leads to minor quantitative errors. 

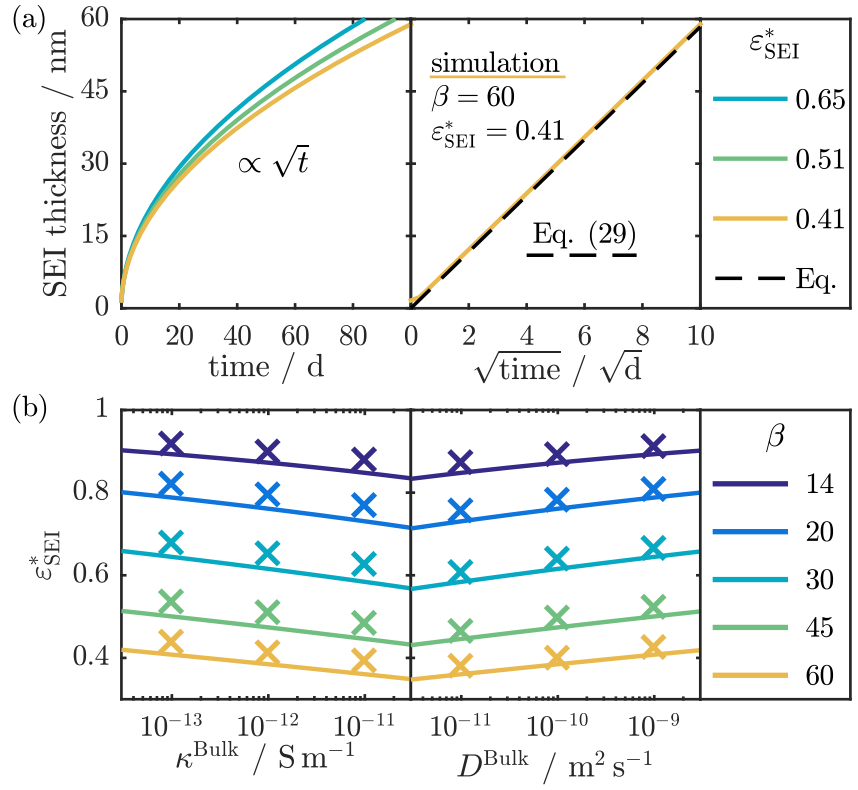

Figure 5. (a) SEI thickness in nm over time $t$ (left) and $\sqrt{t}$ (right) to illustrate the square root of time dependence. The porosity dependence of $L(t)$ is shown on the left side, on the right the simulation is compared to Eq. 29 (dashed line). (b) SEI volume fraction dependence on transport parameters $\kappa$ (left) and $D$ (right). Crosses mark points measured in simulations, lines show predictions by Eq. 34 .

The charge which is irreversibly consumed by SEI formation is equal to

$$
Q_{\text {irr. }}(t)=\frac{2 \varepsilon_{\mathrm{SEI}}^{*} F}{\bar{V}_{\mathrm{SEI}}} A_{\text {total }}^{\text {Elec. }} L(t)=2 A_{\text {total }}^{\text {Elec. }} \sqrt{\frac{\varepsilon_{\mathrm{SEI}}^{*} F \kappa^{*} \Delta \Phi_{\mathrm{EC}}}{\bar{V}_{\mathrm{SEI}}^{\mathrm{Li} \mathrm{i}_{2} \mathrm{EDC}}}} \sqrt{t},
$$

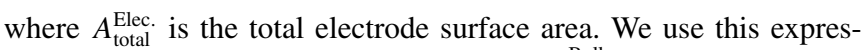
sion, to estimate the electron conductivity $\kappa^{\text {Bulk }}$ by comparing it to capacity fade measurements by Broussely et al. ${ }^{47}$ For this comparison we use $A_{\text {total }}^{\text {Elec. }}=173 \mathrm{~m}^{2}$, taken from ${ }^{31}$ and assume $\varepsilon_{\mathrm{SEI}}^{*}=0.8$. As shown in Figure 6 , we obtain values of $\kappa^{\text {Bulk }}=0.1 \mathrm{pSm}^{-1}$ to $\kappa^{\text {Bulk }}=0.65 \mathrm{pSm}^{-1}$ for $T=30^{\circ} \mathrm{C}$ and $T=60^{\circ} \mathrm{C}$ respectively. These values agree with our previous parameterization. ${ }^{26}$ The corresponding SEI thicknesses equal 50 and $125 \mathrm{~nm}$ after 450 days of storage.

SEI porosity.-As mentioned above, porosity inside the SEI remains constant in time. Further EC reduction stops because the con-

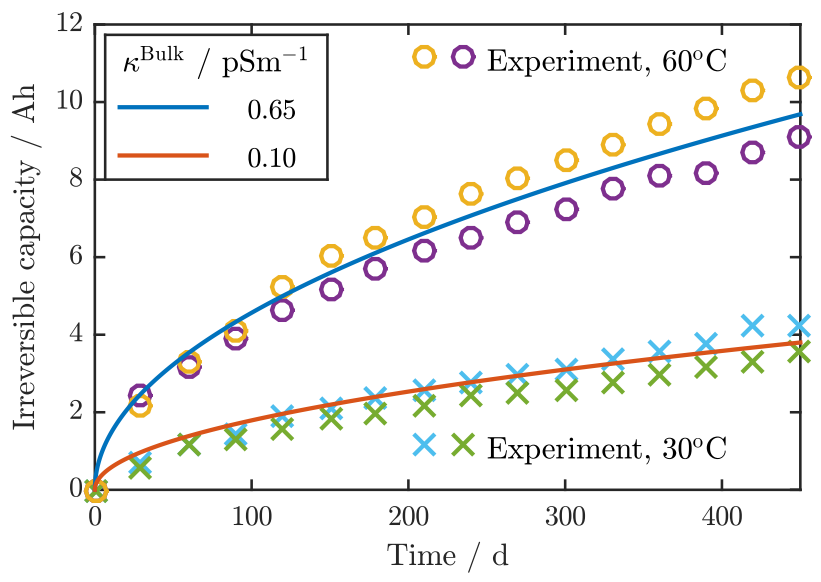

Figure 6. Capacity fade according to Eq. 30 (lines) compared to experimental data (circles and crosses). ${ }^{47}$ centration of the active solvent (EC) is too low and pores are filled with inert co-solvent, see Fig. 4c. Diffusion fluxes of active solvent into the pores are suppressed by the small effective diffusion coefficient $D^{*}=\varepsilon^{* \beta} D^{\text {Bulk }}$. The formation reactions are distributed over the front and cease on its left edge, where porosity equals $\varepsilon^{*}$. Here solvent cannot be supplied at the same rate at which electrons reach the front, forcing the SEI to expand laterally. Consequently, the porosity observed in our simulations depends on the transport parameters of the electrolyte $\left(D, \beta, c_{\mathrm{EC}}^{0}\right)$. All parameters appearing in Eq. 29 determine the speed of SEI expansion and influence porosity as well.

We derive an analytic expression to understand the dependence of the mean SEI porosity $\varepsilon^{*}$ on these parameters. This requires to apply some simplifications to the model equations. First and foremost, reaction kinetics are assumed to be infinitely fast. This has almost no influence on our results because SEI growth is limited by transport and not by reaction kinetics. When the reactions are sufficiently fast, the overpotential $\eta$ is small enough to justify the approximation $\eta_{\mathrm{EC}}=0$ which implies (see Eq. 12a)

$$
c_{\mathrm{EC}}=c_{\mathrm{EC}}^{0} e^{\frac{F\left(\Phi-\Phi_{\mathrm{EC}}^{0}\right)}{2 R T}}, c_{\mathrm{EC}, x}^{\prime}=\frac{c_{\mathrm{EC}} F}{2 R T} \Phi_{x}^{\prime},
$$

where $X_{y}^{\prime}$ denotes the partial derivative $\frac{\partial X}{\partial y}$. Secondly, we simplify our principal equations by neglecting convection. This yields

$$
\begin{gathered}
\frac{\partial \varepsilon}{\partial t}=-\bar{V}_{\mathrm{SEI}}^{\mathrm{Li}_{2} \mathrm{EDC}} A \Gamma r_{\mathrm{EC}}, \\
\frac{\partial \varepsilon c_{\mathrm{EC}}}{\partial t}=\frac{\partial}{\partial x} D c_{\mathrm{EC}, x}^{\prime}-2 A \Gamma r_{\mathrm{EC}}, \\
0=\frac{\partial}{\partial x} \frac{\kappa}{F} \Phi_{x}^{\prime}+2 A \Gamma r_{\mathrm{EC}} .
\end{gathered}
$$

Finally, we analyze Eq. $32 \mathrm{~b}$ in the stationary regime $\left(\partial_{t} \varepsilon c_{\mathrm{EC}} \approx 0\right)$ because porosity and concentration changes in time are small. By summing Equations $32 \mathrm{~b}$ and $32 \mathrm{c}$ while using Eq. 31 to express $c_{\mathrm{EC}, x}^{\prime}$ with $\Phi_{x}^{\prime}$, we obtain

$$
0=\frac{\partial}{\partial x}(\underbrace{\frac{c_{\mathrm{EC}} F D}{2 R T}}_{\tilde{D}}+\underbrace{\frac{\kappa}{F}}_{\tilde{\kappa}}) \Phi_{x}^{\prime} .
$$

Integration yields an expression for $\Phi_{x}^{\prime}$, relating it to the local transport parameters

$$
\Phi^{\prime}=\frac{\xi}{\tilde{D}+\tilde{\kappa}} \text {, resulting in, } \Phi^{\prime \prime}=-\frac{\tilde{\mathrm{D}}_{\varepsilon}^{\prime}+\tilde{\kappa}_{\varepsilon}^{\prime}}{\tilde{\mathrm{D}}+\tilde{\kappa}} \varepsilon_{x}^{\prime} \Phi_{x}^{\prime}-\frac{\tilde{\mathrm{D}}_{c}^{\prime}}{\tilde{\mathrm{D}}+\tilde{\kappa}} \frac{c_{\mathrm{EC}} \Phi_{x}^{\prime 2}}{2 R T},
$$

where $\xi$ is an integration constant. When the SEI is sufficiently thick, we can neglect terms scaling with $\Phi_{x}^{\prime 2}$ because $\Phi_{x}^{\prime}$ is proportional to $L^{-1}$. Using this approximation in Equation 32c after inserting 32a yields

$$
\begin{aligned}
\frac{\partial \varepsilon}{\partial t} & =\frac{\bar{V}_{\mathrm{SEI}}^{\mathrm{Li}_{2} \mathrm{EDC}}}{2} \frac{\partial}{\partial x} \tilde{\kappa} \Phi_{x}^{\prime} \\
& =\frac{\bar{V}_{\mathrm{SEI}}^{\mathrm{Li}_{2} \mathrm{EDC}}}{2}\left(\tilde{\kappa}_{x}^{\prime} \Phi_{x}^{\prime}+\tilde{\kappa} \Phi_{x x}^{\prime \prime}\right) \\
& =\frac{\bar{V}_{\mathrm{SEI}}^{\mathrm{Li}_{2} \mathrm{EDC}}}{2} \frac{\tilde{D} \tilde{\kappa}_{\varepsilon}^{\prime}-\tilde{\kappa} \tilde{D}_{\varepsilon}^{\prime}}{\tilde{D}+\tilde{\kappa}} \varepsilon_{x}^{\prime} \Phi_{x}^{\prime} .
\end{aligned}
$$

We now trace the porosity at a point co-moving with the left edge of the SEI front, see Fig. 3. Here the porosity changes in time according to

$$
\frac{d \varepsilon(L(t), t)}{d t}=\varepsilon_{x}^{\prime} \frac{\partial L}{\partial t}+\frac{\partial \varepsilon}{\partial t} \approx \frac{\bar{V}_{\mathrm{SEI}}^{\mathrm{Li}_{2} \mathrm{EDC}} \varepsilon_{x}^{\prime} \Phi_{x}^{\prime}}{4}\left(\frac{\tilde{\kappa}}{\varepsilon_{\mathrm{SEI}}}+\frac{\tilde{D} \tilde{\kappa}_{\varepsilon}^{\prime}-\tilde{\kappa} \tilde{D}_{\varepsilon}^{\prime}}{\tilde{D}+\tilde{\kappa}}\right),
$$

where the approximation for the thickness evolution Eq. 29 is used. The porosity at this location has an attractive point. This means that 
$\varepsilon$ will converge toward this value in time. This stationary solution equals the mean SEI porosity $\varepsilon^{*}$ which satisfies

$$
\frac{\tilde{\kappa}^{*}}{\tilde{D}^{*}}=\frac{\kappa^{*}}{D^{*}} \frac{2 R T}{c_{\mathrm{EC}} F^{2}}=\frac{1}{2}+\frac{\beta \varepsilon_{\mathrm{SEI}}^{*}}{\varepsilon^{*}} .
$$

We compare this expression to simulation results in Figure 5b. It describes the dependence of porosity on the transport parameters $\kappa^{\text {Bulk }}$, $D^{\text {Bulk }}$ and $\beta$ extremely well. There is a small offset between the SEI porosity determined by the simulation and the analytic prediction. We attribute this to the simplifications made in the derivation of Eq. 34. As we neglect electrolyte convection, the porosity predicted is slightly too low. Much better agreement is found, when the active solvent concentration is low and the influence of solvent convection is small.

In summary, we predict a finite SEI porosity which we propose to measure in appropriate in-situ imaging studies. This prediction assumes long-term storage, consequently all samples need to be stored for an appropriate time span before the measurement. Unfortunately, we cannot quantitatively predict $\varepsilon^{*}$ because it depends strongly on $\beta$, an unknown parameter, see Fig. 5b. Assuming Bruggeman coefficients between $\beta=5$ and $\beta=20$ results in porosities between $\varepsilon^{*}=0.002$ and $\varepsilon^{*}=0.2$.

Neutral lithium interstitial diffusion.-In the simulations discussed above, electron conduction is the rate-limiting transport mechanism. Electron conduction is the most prominent among several transport mechanisms in the solid SEI phase suggested in the literature. ${ }^{18,27,28}$ The findings for the reference scenario discussed in this section, however, are independent of the specific charge transport mechanism. In the following, we demonstrate this by replacing electron conduction with diffusion of neutral lithium interstitials. The latter mechanism is proposed as a potentially rate-limiting mechanism by Shi et al. ${ }^{18}$

We add a mass balance equation for the neutral lithium interstitial concentration $c_{\mathrm{Li}}$

$$
\frac{\partial \varepsilon_{\mathrm{SEI}} c_{\mathrm{Li}}}{\partial t}=-\operatorname{div} j_{\mathrm{D}, \mathrm{Li}}+\dot{n}_{\mathrm{Li}}
$$

where the diffusive flux $j_{\mathrm{D}, \mathrm{Li}}$ has the same porosity dependence as the migration flux in our standard case, see Eq. 19. This transport equation replaces Eq. 8, which describes electron conduction. In this way, we exchange the rate-limiting transport mechanism.

SEI profiles obtained using this mechanism share the same features as those generated with the conduction type mechanism, see Fig. 4a. Again, we observe the formation of a layer with nearly constant porosity. Similar to above, the thickness evolution follows a $\sqrt{t}$ law. Analytic expressions for the thickness evolution and the porosity can be derived analogously to Eq. 29 and Eq. 34, respectively. In conclusion, SEI thickness evolution and porosity are not sufficient to distinguish between these two charge transport mechanisms in the solid SEI phase. Therefore, we study further SEI quantities in next sections.

Additionally, we find that the interstitial concentrations found by Shi et al. ${ }^{18}$ are insufficient to drive SEI formation at a reasonable rate. For the simulation depicted in Fig. 4 a we have used the proposed $\approx 10^{7}$ interstitials $/ \mathrm{cm}^{3}$. To obtain reasonable growth rates we used an the extremely high bulk diffusion coefficient of $0.002 \mathrm{~cm}^{2} / \mathrm{s}$. Alternatively, we obtain reasonable diffusion coefficients for a higher interstitial concentration. Such a concentration would correspond to a smaller interstitial formation energy, approximately 200-300 meV below the value from Shi et al. ${ }^{18}$

Dual-layer SEI.-It is well-known that the SEI is not chemically homogeneous. Therefore, as the next step, we extend the reference scenario by taking a second SEI compound into account. This compound is either produced by co-solvent reduction (II) or by conversion of $\mathrm{Li}_{2} \mathrm{EDC}$ (III). The onset potential for these reactions is chosen as $\Phi_{\mathrm{DMC}}^{0}=\Phi_{\mathrm{Li} \text { EDC }}^{0}=0.3 \mathrm{~V}$ and is below the reduction potential of EC of $0.8 \mathrm{~V}$. In these scenarios, dual-layer structures emerge, as shown in Fig. 7. Depending on the reaction type, the two layers differ in

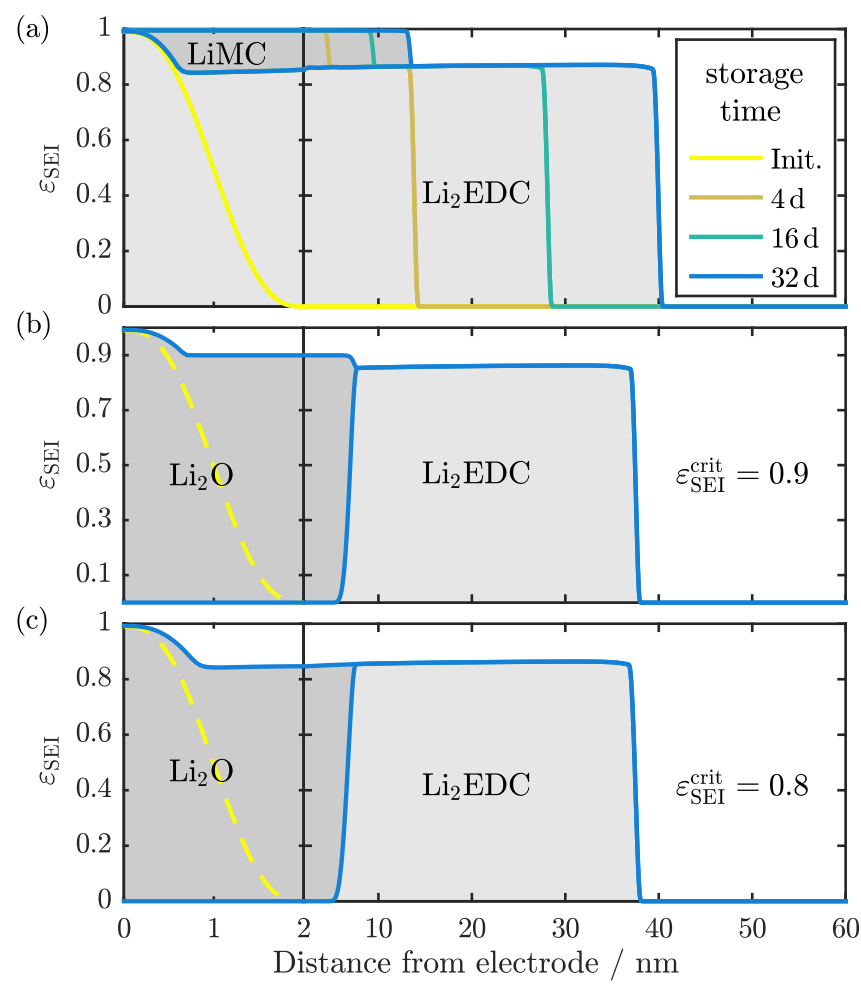

Figure 7. (a) SEI volume fraction evolution with active co-solvent. (b) and (c) show the SEI volume fraction of a dual layer SEI formed with inert cosolvent and unstable $\mathrm{Li}_{2}$ EDC. These simulations differ in the choice of $\varepsilon_{\mathrm{SEI}}^{\mathrm{crit}}$, see Fig. 2.

chemistry, morphology, or both. The total SEI thickness evolves as in the reference scenario. Both layers grow simultaneously and each layer has its own front where the corresponding formation reaction takes place.

Co-solvent reduction.-The volume fraction evolution of a simulation with reacting co-solvent is shown in Fig. 7a. EC reduction proceeds as described in our reference scenario, creating a porous layer of $\mathrm{Li}_{2} \mathrm{EDC}$ (see Fig. 4). Additionally, co-solvent is reduced at the front of the inner layer, filling the pores of the outer layer with LiMC. Co-solvent reduction stops when the layer is dense. It is suppressed because the specific surface area vanishes when $\varepsilon \rightarrow 0$, see Eq. 21. Therefore, a dense layer forms next to the electrode while the outer layer remains porous. $\mathrm{Li}_{2} \mathrm{EDC}$ and $\mathrm{LiMC}$ are both present in the dense layer.

Volume mismatch between the products and reactants of the second reduction reaction induces a convective flow of the electrolyte. This flow carries additional solvent across the SEI front. In turn, the mean porosity of the outer layer $\varepsilon^{*}$ decreases and the SEI becomes denser compared to simulations with inert co-solvent, see Fig. 4a. Therefore, our analytic expression for the porosity Eq. 34 does not predict the porosity of the outer layer as accurately as before.

Conversion Reaction.-The SEI remains to be composed of two layers if co-solvent reduction (II) is replaced with the conversion reaction (III), see Figure $7 \mathrm{~b}$ and $7 \mathrm{c}$. Again, the outer layer is porous and consists of $\mathrm{Li}_{2} \mathrm{EDC}$. The inner layer is created by the conversion of $\mathrm{Li}_{2} \mathrm{EDC}$ and constantly grows at its front. In this case, each layer consists of the products of a single reaction. Compared to simulations with active co-solvent, products of different reduction reactions are no longer mixed in the inner layer.

The porosity of the inner layer depends on the choice of $\alpha\left(\varepsilon_{\mathrm{SEI}}\right)$ (see Eq. 16) or $\varepsilon_{\mathrm{SEI}}^{\text {crit }}$ specifically. As described in the Model section, $\varepsilon_{\mathrm{SEI}}^{\text {crit }}$ determines how dense the SEI can become from accumulation of excess volume by conversion reactions. Here we can distinguish two cases. In Fig. $7 b$ the critical SEI volume fraction $\varepsilon_{\text {SEI }}^{\text {crit }}$ exceeds 

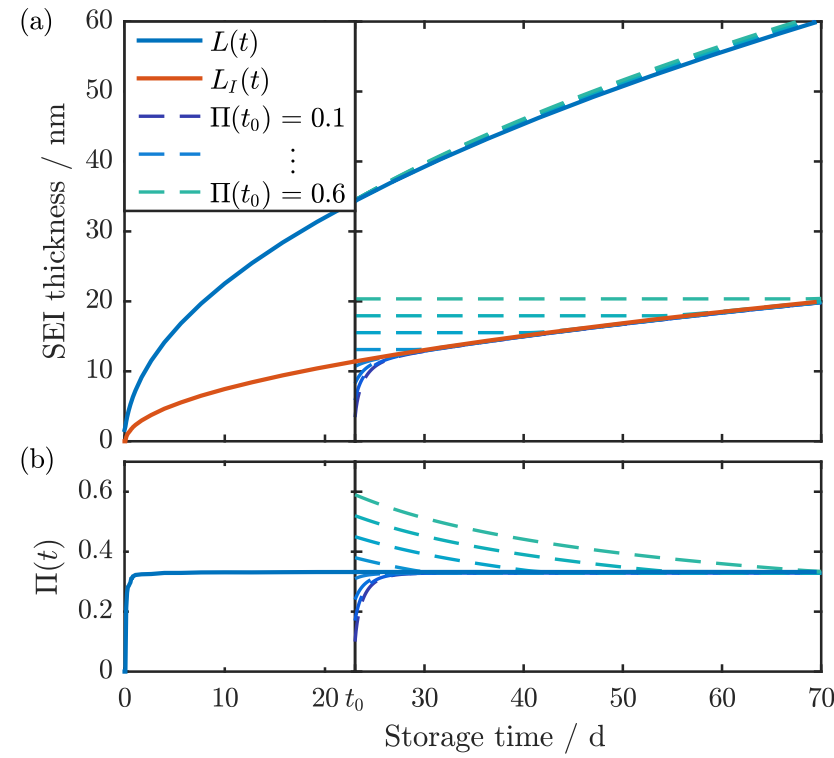

Figure 8. (a) Thickness evolution of the inner and the outer layer in a simulation with active co-solvent (solid lines) compared to numerical solutions of Eqs. 36 (dashed lines). The latter are initialized at $t_{0}=30 \mathrm{~d}$ with different values of $L_{I}\left(t_{0}\right)$. (b) Evolution of $\Pi(t)$ for both simulation and numerical solutions.

the volume fraction of the outer layer. Therefore, excess volume of the conversion reaction can accumulate locally until the SEI volume fraction has reached this value $\varepsilon_{\text {SEI }}^{\text {crit }}$. Further conversion reactions only induce solid convection, thus increasing the thickness of the inner layer and displacing the outer one. The porosity profile shown in Fig. $7 \mathrm{c}$ is created when $\varepsilon_{\mathrm{SEI}}^{\text {crit }}$ is smaller than or equal to the volume fraction of the outer layer. In this case, both layers have the same porosity.

Thickness evolution.-We now discuss the thickness evolution of the dual-layer films. In Fig. 8a, we plot the thickness of the inner layer and the total SEI thickness against the storage time (solid lines). Both layers grow with the square-root of time. In this figure and the subsequent discussion, the inner layer is formed by products of cosolvent reduction (II). For conversion reactions (III), the situation is qualitatively identical.

Analogously to Eq. 29, we derive analytic expressions for the thickness evolution of the dual-layer system. The derivation below is performed for a system with active co-solvent and the index I/O is used for the inner/outer layer respectively, $\left(L_{\mathrm{I}}+L_{\mathrm{O}}=L\right)$. We couple the electronic current in each layer to its growth rate,

$$
\begin{gathered}
\frac{\partial L}{\partial t}=-\frac{\bar{V}_{\mathrm{SEI}}^{\mathrm{Li}_{2} \mathrm{EDC}}}{2 \varepsilon_{\mathrm{SEI}}^{*} F} j_{\mathrm{E}, \mathrm{O}}, \\
\frac{\partial L_{\mathrm{I}}}{\partial t}=-\frac{\bar{V}_{\mathrm{SEI}}^{\mathrm{LiMC}}}{\varepsilon^{*} F}\left(j_{\mathrm{E}, \mathrm{I}}-j_{\mathrm{E}, \mathrm{O}}\right) .
\end{gathered}
$$

We proceed to solve these equations by deriving simple expressions for the current densities in both layers $j_{\mathrm{E}, i}$. To this aim, we discuss the shape of the electric potential $\Phi(x)$ in the SEI. As explained above, reactions occur at the layer fronts only and $\Phi(x)$ increases linearly in each layer. Additionally, porosity and conductivity are constant in each layer. We hold the electrode potential constant $\Phi(x=0)=\Phi_{\mathrm{E}}$. At the SEI front, the potential is given by $\Phi(L) \approx \Phi_{\mathrm{EC}}^{0}$.

We use this to approximate $j_{\mathrm{E}, i}$, similar to Eq. 27

$$
j_{\mathrm{E}, \mathrm{O}}=-\kappa^{*} \frac{\Phi_{\mathrm{EC}}^{0}-\Phi\left(L_{\mathrm{I}}\right)}{L-L_{\mathrm{I}}}, j_{\mathrm{E}, \mathrm{I}}=-\kappa^{\mathrm{Bulk}} \frac{\Phi\left(L_{\mathrm{I}}\right)-\Phi_{\mathrm{E}}}{L_{\mathrm{I}}} .
$$

If the inner layer grows, it holds

$$
\Phi\left(L_{\mathrm{I}}\right)=\Phi_{\mathrm{DMC}}^{0} .
$$

Otherwise, we have to take into account the irreversibility of SEI formation and demand $j_{\mathrm{E}, \mathrm{I}}=j_{\mathrm{E}, \mathrm{O}}$.

$$
\Phi\left(L_{\mathrm{I}}\right)=\left(\Phi_{\mathrm{EC}}-\Phi_{\mathrm{E}}\right)\left(1+\frac{L_{\mathrm{O}}}{L_{\mathrm{I}}} \frac{\kappa^{\mathrm{Bulk}}}{\kappa^{*}}\right)^{-1}+\Phi_{\mathrm{E}} .
$$

$\Phi\left(L_{\mathrm{I}}\right)$ is the maximum of $\Phi_{\mathrm{DMC}}^{0}$ and this modified expression. To conclude, Eq. 36 together with Eq. 37 is a coupled ODE for $L$ and $L_{\mathrm{I}}$ which describes the thickness evolution of each layer.

According to Eq. 36, the growth rate is determined by the same parameters as in simulations with inert co-solvent. These are the conductivity $\kappa^{\text {Bulk }}$, the molar volumes of SEI compounds $\bar{V}_{\mathrm{SEI}}^{i}$ and the applied potential $\Phi_{\mathrm{E}}$. Additionally, the porosity of the outer layer $\varepsilon_{\mathrm{SEI}}^{*}$ appears as an indirect parameter which has to be assumed or measured.

Fig. 8a compares the thickness evolution from numerical solutions of Eqs. 36 (dashed) to a simulation of the full model (solid lines). The figure shows several solutions with different initializations i.e. $L_{\mathrm{I}}\left(t_{0}\right)$ is varied while $L\left(t_{0}\right)$ is fixed. When the initial values match the full simulation, very good agreement is observed and both layers grow with $\sqrt{t}$. The other curves show how the dual layer system reacts to a different initialization. Fig. $8 \mathrm{~b}$ shows how the ratio of the inner to the total SEI thickness

$$
\Pi(t)=L_{I}(t) / L(t), \quad \Pi \in[0,1],
$$

evolves in time. This ratio quickly attains the stationary value $\Pi_{\text {stat }}$ and then remains constant for the rest of the simulation. In a real battery, $\Pi$ can deviate from this stationary value if the electrode potential is varied or if the SEI is physically damaged. As illustrated in Fig. 8b, $\Pi_{\text {stat }}$ is a stationary point of $\Pi(t)$ and satisfies

$$
\frac{\partial \Pi}{\partial t}=\frac{\partial_{t} L_{I}}{L}-\frac{L_{I} \partial_{t} L}{L^{2}} \stackrel{!}{=} 0 \rightarrow L \partial_{t} L_{I}=L_{I} \partial_{t} L .
$$

With Eqs. (36) we can rearrange this condition into a quadratic equation in $\Pi_{\text {stat }}$

$$
\frac{\bar{V}_{\mathrm{SEI}}^{\mathrm{Li} \mathrm{EDC}}}{2 \bar{V}_{\mathrm{SEI}}^{\mathrm{LiMC}}} \varepsilon^{*} \Pi_{\mathrm{stat}}^{2}+\left(1+\frac{\Delta \Phi_{\mathrm{DMC}}}{\varepsilon_{\mathrm{SEI}}^{* 1.5} \Delta \Phi_{\mathrm{diff}}}\right) \Pi_{\text {stat }}+\frac{\Delta \Phi_{\mathrm{DMC}}}{\varepsilon_{\mathrm{SEI}}^{* 1.5} \Delta \Phi_{\mathrm{diff}}}=0 .
$$

$\Pi_{\text {stat }}$ is the positive solution of this expression. It depends most strongly on the electrode potential $\Phi_{\mathrm{E}}$ and the onset potential $\Phi_{i}^{0}$ of each reduction reaction.

The stationary value is attained after long-term storage with constant electrode potential. When the electrode potential is changed, $\Pi$ will deviate from the new stationary ratio. Then, further SEI growth will be distributed such that this new stationary value $\Pi_{\text {stat }}$ is attained. This process is fast (1-2 days) when $\Pi<\Pi_{\text {stat }}$ as illustrated in Fig. $8 \mathrm{~b}$. In this case, the inner layer needs to become thicker. The rate at which $\Pi$ converges toward $\Pi_{\text {stat }}$ is slow, when $\Pi>\Pi_{\text {stat }}$ because the inner layer cannot decrease its thickness. Instead, the outer layer needs to grow to restore $\Pi_{\text {stat }}$. This takes longer, in part due to the stoichiometry of both reduction reactions. Furthermore, electrons need to traverse a longer distance to reach the front of the outer layer.

By using the relation $L_{I}=\Pi_{\text {stat }} L$, we can solve Eq. 36a and obtain an analytical expression for the thickness evolution

$$
L(t)=\sqrt{\frac{\kappa^{*} \bar{V}_{\mathrm{SEI}}^{\mathrm{Li}_{2} \mathrm{EDC}}}{\varepsilon_{\mathrm{SEI}}^{*} F} \frac{\Delta \Phi_{\mathrm{diff}}}{1-\Pi_{\mathrm{stat}}}} \sqrt{t} .
$$

Most formulas in this section are not valid if the inner layer is formed by a conversion reaction. For this system, a few changes need to be made in the derivation above. However, these changes do not alter the results in a qualitative way. This means that all results above can be transferred. Eq. 41 remains valid if the correct value of $\Pi_{\text {stat }}$ is used. The only noteworthy quantitative difference is the rate at which $\Pi(t)$ converges toward the stationary value. This process is now slower when $\Pi<\Pi_{\text {stat }}$ because more electrons are needed to expand the inner layer.

We highlight that the SEI dual-layer structure should be observable in long-term storage experiments, e.g., in neutron-scattering imaging. 
Charge vs. solvent transport.-In the preceding sections, we discuss scenarios in which charge transport in the solid SEI is the ratelimiting transport mechanism. Even though we model solvent diffusion through the SEI pores, electrolyte transport does not become rate-limiting. This is due to the low porosity of the SEI formed in our simulations which makes solvent diffusion slower than charge transport. Now we discuss how structural properties may prevent the formation of a dense SEI. In this scenario, solvent diffusion inside the SEI becomes faster, potentially making solvent diffusion the ratelimiting transport mechanism. Hence we can study how SEI grows for different rate-limiting transport mechanisms.

Structural properties can emerge from surface tensions in the porous structure which can influence SEI morphology and porosity. In this way, the interplay of surface energy and packing structure results in a minimum porosity. Alternatively, small SEI particles could have a certain tightest packing. In both cases SEI will accumulate locally until this porosity is reached, then reactions will displace existing particles instead of further decreasing the porosity. This effect is reminiscent of the solid convection defined in the Model section. Note that it requires deeper insights into the chemistry and structure of the composite solid SEI material to accurately determine the minimum porosity.

Similar to our reference scenario, we consider the reduction of EC and assume that the co-solvent is inert. This reaction is coupled to the solid convection velocity

$$
\varepsilon_{\mathrm{SEI}} \operatorname{div} \tilde{v}=\alpha\left(\varepsilon_{\mathrm{SEI}}\right) \cdot \bar{V}_{\mathrm{SEI}}^{\mathrm{Li}_{2} \mathrm{EDC}} \cdot \Gamma A \cdot r_{\mathrm{EC}} .
$$

Here, $\alpha\left(\varepsilon_{\text {SEI }}\right)$ models a smooth transition from local accumulation to SEI displacement, see Fig. 2a. As elaborated on in the Model section, this transition takes place at the SEI volume fraction $\varepsilon_{\mathrm{SEI}}^{\text {crit }}$, a new parameter of our model. Therefore, Eq. 42 will prevent the SEI volume fraction to exceed $\varepsilon_{\mathrm{SEI}}^{\text {crit }}$.

Now, charge transport and solvent transport compete. The mean solid volume fraction $\varepsilon_{\mathrm{SEI}}^{*}$ inside the SEI determines the rate-limiting transport mechanism. We distinguish two cases by comparing $\varepsilon_{\mathrm{SEI}}^{\text {crit }}$ with the stationary solution $\varepsilon_{\text {SEI }}^{\text {stat }}$ of Eq. 34 .

1. For $\varepsilon_{\mathrm{SEI}}^{\text {crit }}>\varepsilon_{\mathrm{SEI}}^{\text {stat }}$, i.e., large SEI volume fractions, SEI formation is not disturbed and proceeds exactly like in our reference scenario. In this case, electron conduction is rate-limiting and the porosity establishes itself through a balance between growth and transport at the SEI front, see Eq. 34. Our model does not allow for a denser structure for a given parameter set.

2. For $\varepsilon_{\mathrm{SEI}}^{\text {crit }}<\varepsilon_{\mathrm{SEI}}^{\text {stat }}$, i.e., small SEI volume fractions, solid convection (42) limits the SEI volume fraction. In this case, the mean SEI volume fraction is decreased $\varepsilon_{\mathrm{SEI}}^{*}=\varepsilon_{\mathrm{SEI}}^{\text {crit }}$ and the SEI porosity is increased. Therefore, solvent diffusion through the SEI pores is accelerated and can become rate-limiting.

In summary, structural properties can only increase the mean porosity $\varepsilon^{*}$ and accelerate solvent diffusion.

Our extended model captures the two growth mechanisms studied in the literature. SEI can form at the electrode/SEI interface limited by solvent/salt diffusion through the SEI. ${ }^{30,31}$ Additionally, SEI can form at the SEI front limited by charge transport through the SEI. ${ }^{26-29}$ Below we will proof this correlation between rate-limiting transport mechanism and reaction interface position. We are the first to find an intermediate regime where the reaction interface is located inside the SEI as depicted in Fig. 9. In this case, both mechanisms contribute to the formation rate and the SEI is divided by the reaction interface (marked red). In the inner region, electrons migrate away from the electrode, whereas solvent molecules diffuse toward the electrode in the outer one.

Now, we calculate the relative location $\tilde{\Pi}=L_{\text {reaction }} / L$ of this interface. Electron and solvent transport to this location are balanced and supply the reaction

$$
2 j_{\mathrm{E}}=2 F j_{\mathrm{D}, \mathrm{EC}} .
$$

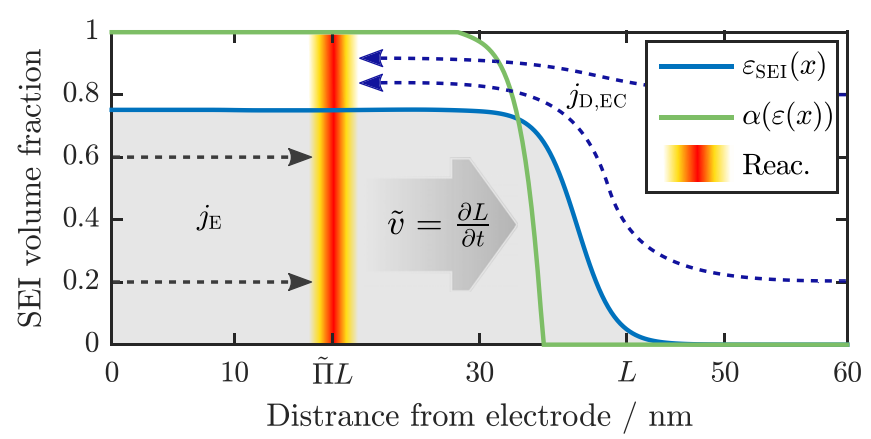

Figure 9. Schematic of self-shaping SEI growth with $\tilde{\Pi} \approx 0.4$. The reaction interface (marked red) is inside the SEI and the reaction is "fueled" by an electronic current $j_{\mathrm{E}}$ and a diffusion current of solvent $j_{\mathrm{D}, \mathrm{EC}}$. SEI compounds which form at this interface do not decrease the porosity, because $\alpha\left(\varepsilon_{\text {SEI }}(\tilde{\Pi} L)\right)=1$. Instead, they induce the convection velocity $\tilde{v}$ which causes SEI growth.

We can approximate each flux by assuming constant porosity and a linear progression of potential and concentration within the SEI

$$
\frac{\kappa^{*} \Delta \Phi_{\mathrm{EC}}}{\tilde{\Pi} L} \approx \frac{F D^{*} c_{\mathrm{EC}}}{(1-\tilde{\Pi}) L}, \rightarrow \tilde{\Pi}=\frac{\kappa^{*} \Delta \Phi_{\mathrm{EC}}}{D^{*} F c_{\mathrm{EC}}+\kappa^{*} \Delta \Phi_{\mathrm{EC}}} .
$$

The ratio $\tilde{\Pi}$ is independent of SEI thickness $L$. It quantifies the relative share of electron conduction on the rate-limiting role. Fig. 10 shows, how $\tilde{\Pi}$ depends on the effective transport parameters $\kappa^{*}$ and $D^{*}$. $\tilde{\Pi} \approx 1$ if electron conduction is the rate-limiting transport mechanism, $\kappa^{*} \Delta \Phi_{\mathrm{EC}} \gg D^{*} F c_{\mathrm{EC}}$ (top-left). Solvent diffusion is the rate-limiting transport mechanism if $\tilde{\Pi} \approx 0, \kappa^{*} \Delta \Phi_{\mathrm{EC}} \ll D^{*} F c_{\mathrm{EC}}$ (bottom-right). The intermediate regime spans from the bottom-left to the top-right. Here, both transport mechanisms are roughly equally fast, $\kappa^{*} \Delta \Phi_{\mathrm{EC}} \approx$ $D^{*} F c_{\mathrm{EC}}$.

The transition from electron conduction to solvent diffusion limited growth occurs when $\varepsilon_{\mathrm{SEI}}^{\text {crit }}$ becomes smaller than $\varepsilon_{\mathrm{SEI}}^{\text {stat }}$. Based on the

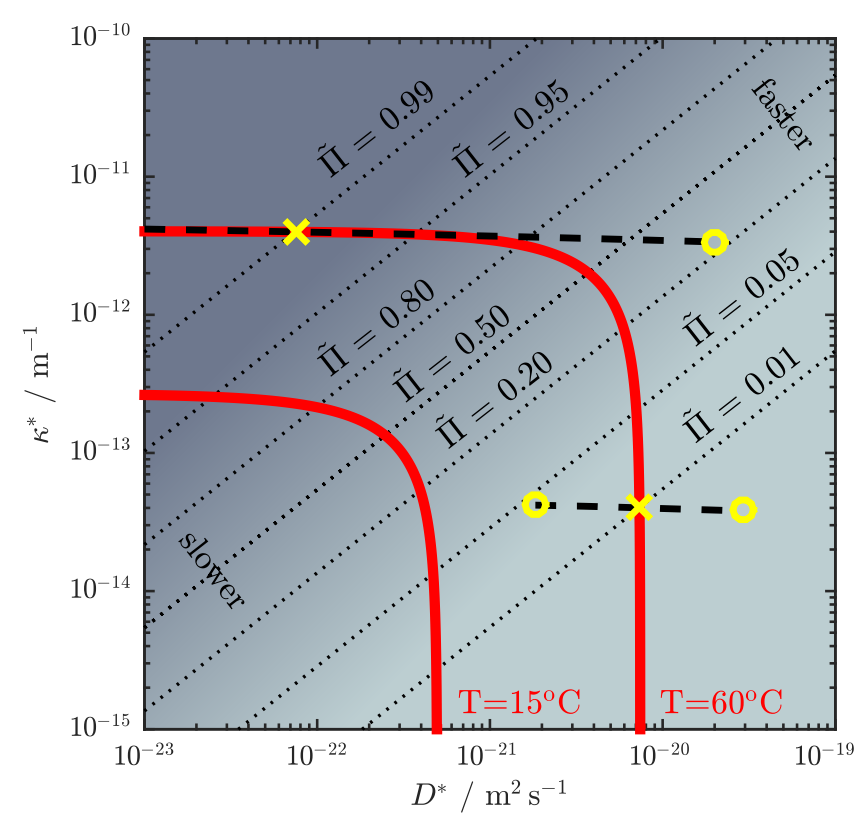

Figure 10. Relative position of the reaction interface $\tilde{\Pi}$ depending on the effective transport parameters $D^{*}$ and $\kappa^{*}$ according to Eq. 43. The red lines show parameter sets with identical SEI growth rates and satisfy Eq. 45. Dashed black lines show how two parameter sets $D^{*} / \kappa^{*}$ (marked with yellow crosses) move when porosity is changed but $\kappa^{\text {Bulk }}$ is fixed. The lines end in yellow circles where the formation rate is double (right) or half (left) of the original growth rate. 
values discussed earlier, we conclude that $0.8<\varepsilon_{\text {SEI }}^{\text {crit }}<0.998$ would be necessary for solvent diffusion limited growth.

Growth rate analysis.-Let us now evaluate the SEI growth rate for this general, mixed growth scenario (see Fig. 9). Based on the dependence of the growth rate on the material parameters, we discuss how observable SEI properties depend on the underlying rate-limiting mechanism. We obtain an analytical expression for the thickness evolution of these SEIs, by exchanging $L$ with $\tilde{\Pi} L$ in the derivation of Eq. 29

$$
\begin{aligned}
L(t) & =\sqrt{\frac{\kappa^{*} \Delta \Phi_{\mathrm{EC}} \bar{V}_{\mathrm{SEI}}^{\mathrm{Li}_{2} \mathrm{EDC}}}{\varepsilon_{\mathrm{SEI}}^{*} F \tilde{\Pi}} \sqrt{t},} \\
& =\sqrt{\frac{\bar{V}_{\mathrm{SEI}}^{\mathrm{Li}_{2} \mathrm{EDC}}}{\varepsilon_{\mathrm{SEI}}^{*} F}\left(\kappa^{*} \Delta \Phi_{\mathrm{EC}}+D^{*} F c_{\mathrm{EC}}\right)} \sqrt{t} .
\end{aligned}
$$

Comparison to Eq. 29 reveals that adding solvent diffusion accelerates SEI formation. The $\sqrt{t}$-growth law is still valid as SEI growth is limited by reactant transport.

Naturally, only a subset of the combinations of $D^{*}$ and $\kappa^{*}$ yields reasonable SEI growth rates. A good measure for the growth rate is

$$
\frac{\partial L^{2}}{\partial t}=\dot{L^{2}}=\frac{\bar{V}_{\mathrm{SEI}}^{\mathrm{Li}_{2} \mathrm{EDC}}}{F \varepsilon_{\mathrm{SEI}}^{*}}\left(\kappa^{*} \Delta \Phi_{\mathrm{EC}}+D^{*} F c_{\mathrm{EC}}\right)
$$

which is constant in time for square-root like growth. In Fig. 10 the red lines correspond to growth rates observed at $T=15 / 60{ }^{\circ} \mathrm{C} .{ }^{26,30,48}$ When moving along one of these lines, $\tilde{\Pi}$ increases monotonically from 0 to 1 . SEI growth is limited by a single transport mechanism, unless both effective transport parameters, $D^{*}$ and $\kappa^{*}$, are finely attuned to one another. These cases ( $\tilde{\Pi} \approx 1$ and $\tilde{\Pi} \approx 0$ ) are recovered, when one of the effective transport parameter vanishes. If $D^{*}$ is small, electron conduction determines the growth rate and $\kappa^{*}$ converges toward the values found in. ${ }^{26}$ If $\kappa^{*}$ is small, solvent diffusion is rate-limiting and $D^{*}$ converges toward values found in Refs. 30,31

At this point, we want to draw first conclusions with respect to the rate-limiting transport mechanism. As discussed earlier, SEI porosity will attain a small value $(0.002$ to 0.2$)$ in our reference scenario, where electron conduction is the rate-limiting transport mechanism. Therefore, the SEI volume fraction is approximately one and the growth rate does not depend strongly on the porosity and the Bruggeman coefficient. Instead it is mostly determined by $\kappa^{\text {Bulk }}$. This is different if solvent diffusion is the rate-limiting. In this case the effective transport parameter scales with $\varepsilon$ (to the power of $\beta$ ) which is close to zero. This means that $D^{*}$ depends strongly on three parameters, namely $\varepsilon_{\mathrm{SEI}}^{\text {crit }}, \beta$ and $D^{\text {Bulk }}$. SEI formation is a common phenomenon in lithium-ion batteries, occurring in many different systems. The different growth rates of these SEIs lie within two orders of magnitude, even when the SEI chemistry is not comparable. This would imply that $\varepsilon_{\mathrm{SEI}}^{\text {crit }}$ and $\beta$ are correlated in some way. However, we cannot find any reason why this should be the case. Therefore, it appears unlikely for solvent diffusion to be the rate-limiting transport mechanism.

We now study this difference from another perspective. To this aim, we use the growth rate $\dot{L}^{2}$ and the relative location of the reaction interface $\tilde{\Pi}$ as parameters to label SEIs (instead of $\kappa^{*}$ and $D^{*}$ ). The variation of the SEI growth rate with respect to small porosity fluctuations $\varepsilon_{\mathrm{SEI}}^{*}$ is equal to

$$
\frac{\partial \dot{L}^{2}}{\partial \varepsilon_{\mathrm{SEI}}^{*}}=\dot{L^{2}}\left[\frac{1.5 \tilde{\Pi}-1}{\varepsilon_{\mathrm{SEI}}^{*}}-\frac{\beta(1-\tilde{\Pi})}{1-\varepsilon_{\mathrm{SEI}}^{*}}\right] .
$$

We now evaluate and compare the relative variation in the growth rate from a small porosity change $\Delta \varepsilon$

$$
\tilde{\Pi} \rightarrow 0 \frac{\Delta \dot{L^{2}}}{\dot{L^{2}}} \approx\left(1+\frac{\beta \varepsilon_{\mathrm{SEI}}^{\tilde{\Pi} \rightarrow 0}}{1-\varepsilon_{\mathrm{SEI}}^{\tilde{\Pi} \rightarrow 0}}\right) \frac{\Delta \varepsilon}{\varepsilon_{\mathrm{SEI}}^{\tilde{\Pi} \rightarrow 0}},
$$

$$
\tilde{\Pi} \rightarrow 1 \frac{\Delta \dot{L^{2}}}{\dot{L^{2}}} \approx \frac{1}{2} \frac{\Delta \varepsilon}{\varepsilon_{\mathrm{SEI}}^{\text {stat }}} .
$$

This variation is much larger if solvent diffusion is the rate-limiting transport mechanism $(\tilde{\Pi} \rightarrow 0)$ because either $\beta$ or $\left(1-\varepsilon_{\mathrm{SEI}}^{*}\right)^{-1}$ is large.

We illustrate this in Fig. 10 where two combinations of $D^{*}$ and $\kappa^{*}$ are marked with a yellow cross. Both SEIs have the same growth rate because they are located on the same red line. The difference between these films is the rate-limiting mechanism facilitating the growth. One is solely governed by electron conduction $(\tilde{\Pi} \approx 1)$ whereas solventdiffusion is limiting the other one $(\tilde{\Pi} \approx 0)$. We now apply a small perturbation $\Delta \varepsilon$ to the porosity of each film. This changes the effective transport parameters $\kappa^{*}$ and $D^{*}$ according to the Bruggeman relation Eq. 18 and Eq. 19 ( $\kappa^{\text {Bulk }}$ is kept constant). The new combination is located on the dashed line in Fig. 10 and has a different growth rate according to Eq. 45 . The black lines end in yellow circles where the growth rate is twice or half as large as the original one. It can be seen that the growth rate is hardly influenced by porosity fluctuations if electron conduction is the rate-limiting transport mechanism $(\tilde{\Pi} \approx 1)$. Here, the dashed line remains close to the red one for small perturbations. Therefore, large porosity fluctuations are necessary to observe a significant change in the growth rate. If solvent diffusion is the rate-limiting transport mechanism $(\tilde{\Pi} \approx 0)$, however, the dashed line is almost orthogonal to the red one. Here, SEI formation is far more susceptible to porosity changes and small fluctuations can alter the growth rate by a factor of two.

Finally, we propose to probe the sensitivity to porosity fluctuations in an experiment and identify the rate-limiting transport mechanism. SEI is deformed during cycling due to volume changes of the electrode particles. These deformations change the porosity which in turn affect the growth rate. This results in systematic variations of SEI thickness when the electrode material deforms anisotropically, e.g., on HOPG. We predict notable thickness differences correlating with the atomistic orientation of the electrode surface.

This could be observed in the imaging experiment proposed earlier in this section. Alternatively, information about the rate-limiting transport mechanism could be obtained in a different experiment. We propose to add additional, marked (e.g. isotopically, see Ref. 7) solvent/electrolyte to a cell with a well-established SEI. The location of newly formed SEI can then be determined with depth profiling techniques after a long storage period.

\section{Discussion}

The quality of theoretical studies depends on reliable parameter choices and model assumptions. In this section, we discuss the validity of our choices. To this aim, we justify our assumptions and discuss the dependence of our predictions on them. Our model relies on two important assumptions. Firstly, we assume that the SEI is homogeneous parallel to the electrode surface and develop a one dimensional model. Secondly, we choose a specific dependence of transport parameters on porosity. Besides these assumptions, we make use of physical restrictions such as mass, volume, and charge conservation.

Most obvious, SEI thickness, see Eq. 29, and porosity, see Eq. 34, strongly depend on transport parameters $\left(\kappa^{\text {Bulk }}, D^{\text {Bulk }}, \beta\right)$ as discussed above. SEI porosity, for example, is governed by the Bruggeman coefficient $\beta$ of the electrolyte. Thermodynamic parameters, such as the density of SEI compounds and the onset potential of reduction reactions influence our results as well. However, unlike transport parameters we know these parameters reasonably well. Therefore, an inaccurate choice of these thermodynamic parameters does not influence our results in a significant way. The kinetics of the fast reduction reactions characterized by the activation energy $E_{\mathrm{A}}$ are not critical as well. The only exception is the onset potential of the second reduction reaction. This parameter strongly influences the thickness of the inner layer. Nevertheless, the qualitative observations of the dual-layer systems remain unchanged.

An assailable model assumption is the use of conventional electron conduction in SEI compounds. It is known that several common SEI 
compounds have large bandgaps, i.e. $\mathrm{Li}_{2} \mathrm{EDC},{ }^{21} \mathrm{Li}_{2} \mathrm{CO}_{3}{ }^{18}$ and $\mathrm{LiF}^{49}$ Corresponding conductivities are well below the values which are necessary to drive long term SEI formation at realistic rates. Nevertheless, SEI composition is diverse and a conduction like mechanism could emerge. This could be due to defects or band-bending on grain boundaries inside the SEI. Interface effects on such boundaries can promote lithium-ion and potentially electron mobility as shown by Zhang et al. ${ }^{20}$ We highlight that the specific transport mechanism used does as demonstrated by replacing conduction with neutral lithium interstitial diffusion. Any mechanism which transports charges though the SEI for the reduction of the solvent at the SEI/electrolyte interface will produce qualitatively similar results. The only requirement is that the mechanism decreases linearly with SEI thickness and that the transport occurs in the solid SEI.

Our assumption of homogeneity parallel to the electrode surface is seemingly contradicted by TEM images of fluctuating SEI thickness. ${ }^{6}$ Such measurements, however, typically relate to initial molecular layers of the SEI which our model does not describe. Furthermore, our model offers three explanations for fluctuations in thickness. Fluctuations in the initial SEI composition might locally affect the conductivity. Alternatively, different electrode surfaces, e.g., the basal/edge planes on graphite, can yield different electron injection rates into the SEI. Lastly, SEI thickness fluctuations are expected if solvent diffusion is the rate-limiting process. Our model remains to be applicable locally if these fluctuations occur on a length scale comparable to the SEI thickness. If SEI is exposed to large mechanical stress, e.g., on silicon electrodes, ${ }^{12}$ local properties dominate SEI evolution and our model cannot be applied.

Finally, we keep our model simple and clear on purpose and neglect a couple of details. For example, we do not take into account dissolution of SEI species ${ }^{4}$ which competes with SEI growth. A nucleation and precipitation process for SEI formation has been proposed by Ushirogata et al. ${ }^{21}$ Nucleation and growth of larger SEI particles in solution might be essential during the formation of the initial SEI. Modeling this process would delay the reaction and the precipitation process, which would not influence the long time SEI growth. We neglect this mechanism because we focus on long-term SEI formation. SEI material lost by diffusion into the bulk electrolyte phase could be accounted for by using an effective stoichiometry for the reduction reaction.

\section{Summary}

In this work, we discuss a novel one-dimensional model which describes long-term SEI growth. ${ }^{26}$ We study several plausible scenarios and predict observable SEI properties depending on the respective assumptions. In all scenarios, SEI thickness evolves with the square root of time because SEI growth is limited by the transport of SEI precursors through the SEI.

In our reference scenario structural properties do not prevent the formation of a dense SEI. Then electron conduction is the rate-limiting transport mechanism. Our model predicts the formation of a porous SEI. SEI porosity is almost constant throughout the film and does not change in time. It is the result of an interplay of two transport processes, electron conduction away from the electrode and solvent diffusion toward the electrode. Therefore, porosity depends solely on the parameters characterizing these processes.

Solvent diffusion is the rate-limiting transport mechanism if structural properties prevent the formation of a dense SEI. We find that the growth rate of the SEI is very susceptible to porosity fluctuations in this case. Therefore, we predict an inhomogeneous thickness distribution of SEI on electrodes with anisotropic volume expansion. If solvent diffusion is the rate-limiting transport mechanism, such fluctuations will be observable in a suitable imaging experiment such as those proposed in the Simulation results section.

Replacing electron conduction with diffusion of neutral lithium interstitials only alters the aforementioned predictions quantitatively. This illustrates that they are universal and independent from the specific transport mechanism in the solid SEI.
In scenarios where two reduction reactions are considered, we observe an additional inner SEI layer close to the electrode. The two layers have different chemical compositions and may also exhibit different morphologies. These properties can be observed and employed to identify the type of the second reduction reaction. We find that the ratio of the inner layer thickness to the total SEI thickness tries to attain a stationary value. This value depends on the electrode potential and will be attained after the electrode potential remained constant for a longer period of time $(\approx 60$ days). It does not change when the SEI ages and is restored when the SEI is physically damaged. Observing such a connection between the thicknesses of inner and the outer layer would suggest electron conduction to be the rate-limiting transport mechanism.

\section{Conclusions}

In this article, we discuss a new model to describe long-term SEI growth on negative electrodes. Our model is the first to capture SEI morphology in a spatially resolved way. Explicitly, we explain the growth of a SEI with finite porosity. We can model different ratelimiting transport mechanisms in the solid SEI phase. Additionally, we can adjust SEI porosity and enable solvent diffusion through the pores to be the rate-limiting transport mechanism. This enables us to predict SEI properties which are unique to each mechanism. These predictions are observable in suitable experiments and should allow to draw conclusions with respect to the rate-limiting transport mechanism for SEI growth. To this aim, we propose in-situ imaging studies of well-established SEI, e.g., with TEM or neutron reflectometry.

\section{Acknowledgments}

This work was supported by the German Federal Ministry of Education and Research (BMBF) in the project Li-EcoSafe (03X4636A). Further support was provided, by the bwHPC initiative and the bwHPC-C5 project through associated compute services of the JUSTUS HPC facility at the University of Ulm.

\section{Appendix}

Table AI. Nomenclature and description of frequent quantities. Parameters are described and given in Table $I$.

\begin{tabular}{|c|c|c|}
\hline & Description & Unit \\
\hline$\varepsilon / \varepsilon_{\text {SEI }}$ & Porosity/volume fraction of the SEI & - \\
\hline$c$ & Main solvent (EC) concentration & $\mathrm{mol} \mathrm{m}^{-3}$ \\
\hline$c_{\mathrm{Li}}$ & Neutral lithium interstitial concentration & $\mathrm{mol} \mathrm{m}^{-3}$ \\
\hline$\Phi$ & Electronic potential (solid SEI) & $\mathrm{Vm}^{-1}$ \\
\hline$v / \tilde{v}$ & Electrolyte/solid convective velocity & $\mathrm{ms}^{-2}$ \\
\hline$j_{\mathrm{E}}$ & Electronic current in the solid SEI phase & $\mathrm{A} \mathrm{m}^{-2}$ \\
\hline$r_{j}$ & Turnover of reaction " $j \rightarrow k$ " & $\mathrm{mol} \mathrm{s}^{-1} \mathrm{~m}^{-3}$ \\
\hline$A$ & Specific surface area of the porous SEI & $\mathrm{m}^{-1}$ \\
\hline$L / L_{I}$ & Thickness of the SEI / inner SEI layer & $\mathrm{nm}$ \\
\hline$\Pi$ & Ratio of $L_{I}$ and $L, \Pi=L_{I} L^{-1}$ & - \\
\hline$\tilde{\Pi}$ & $\begin{array}{l}\text { Location of the reaction interface relative } \\
\text { to } L\end{array}$ & - \\
\hline$\alpha(\varepsilon)$ & $\begin{array}{l}\text { Transition function between local } \\
\text { accumulation and SEI expansion }\end{array}$ & - \\
\hline$\varepsilon^{*} / \varepsilon_{\mathrm{SEI}}^{*}$ & Average SEI porosity/volume fraction & - \\
\hline$D^{*}$ & $\begin{array}{l}\text { Solvent diffusion coefficient at average } \\
\text { SEI porosity } D^{*}=\varepsilon^{* \beta} D^{\text {Bulk }}\end{array}$ & $\mathrm{m}^{2} \mathrm{~s}^{-1}$ \\
\hline$\kappa^{*}$ & $\begin{array}{l}\text { SEI conductivity at average SEI volume } \\
\text { fraction } \kappa^{*}=\varepsilon_{\text {SEI }}^{1.5} \kappa^{\text {Bulk }}\end{array}$ & $\mathrm{S} \mathrm{m}^{-1}$ \\
\hline$\Delta \Phi_{\mathrm{EC}}$ & $\Phi_{\mathrm{EC}}^{0}-\Phi_{\mathrm{E}}$ & $\mathrm{V}$ \\
\hline$\Delta \Phi_{\mathrm{DMC}}$ & $\Phi_{\mathrm{DMC}}^{0}-\Phi_{\mathrm{E}}$ & $\mathrm{V}$ \\
\hline$\Delta \Phi_{\text {diff }}$ & $\Phi_{\mathrm{EC}}^{0}-\Phi_{\mathrm{DMC}}$ & $\mathrm{V}$ \\
\hline
\end{tabular}




\section{References}

1. S. Kang, M. H. Park, H. Lee, and Y. K. Han, Electrochemistry Communications, 23 , 83 (2012).

2. E. Peled, Journal of The Electrochemical Society, 126, 2047 (1979).

3. D. Aurbach, A. Zaban, Y. Ein-Eli, I. Weissman, O. Chusid, B. Markovsky, M. Levi, E. Levi, A. Schechter, and E. Granot, Journal of Power Sources, 68, 91 (1997).

4. D. Aurbach, Journal of Power Sources, 89, 206 (2000).

5. S. H. Kang, D. P. Abraham, A. Xiao, and B. L. Lucht, Journal of Power Sources, 175, 526 (2008).

6. M. Nie, D. P. Abraham, D. M. Seo, Y. Chen, A. Bose, and B. L. Lucht, The Journal of Physical Chemistry C, 117, 25381 (2013).

7. A. L. Michan, M. Leskes, and C. P. Grey, Chemistry of Materials, 28, 385 (2015).

8. K. Xu, Y. Lam, S. S. Zhang, T. R. Jow, and T. B. Curtis, Journal of Physical Chemistry C, 111, 7411 (2007).

9. O. Borodin, M. Olguin, C. E. Spear, K. W. Leiter, and J. Knap, Nanotechnology, 26, $354003(2015)$

10. A. Xiao, L. Yang, B. L. Lucht, S.-H. Kang, and D. P. Abraham, Journal of The Electrochemical Society, 156, A318 (2009).

11. M. Nie and B. L. Lucht, Journal of The Electrochemical Society, 161, 1001 (2014).

12. M. Nie, D. P. Abraham, Y. Chen, A. Bose, and B. L. Lucht, The Journal of Physical Chemistry C, 117, 13403 (2013)

13. C. L. Campion, W. Li, and B. L. Lucht, Journal of The Electrochemical Society, 152 A2327 (2005).

14. S. F. Lux, I. T. Lucas, E. Pollak, S. Passerini, M. Winter, and R. Kostecki, Electrochemistry Communications, 14, 47 (2012).

15. M. Wagner, P. Raimann, A. Trifonova, K.-C. Moeller, J. Besenhard, and M. Winter, Electrochemical and Solid-State Letters, 7, A201 (2004).

16. S. J. Harris and P. Lu, Journal of Physical Chemistry C, 117, 6481 (2013).

17. P. Lu, C. Li, E. W. Schneider, and S. J. Harris, Journal of Physical Chemistry C, 118 896 (2014)

18. S. Shi, P. Lu, Z. Liu, Y. Qi, L. G. Hector, H. Li, and S. J. Harris, Journal of the American Chemical Society, 134, 15476 (2012).

19. O. Borodin, G. R. V. Zhuang, P. N. Ross, and K. Xu, Journal of Physical Chemistry C, 117, 7433 (2013).

20. Q. Zhang, J. Pan, P. Lu, Z. Liu, M. W. Verbrugge, B. W. Sheldon, Y. T. Cheng, Y. Qi, and X. Xiao, Nano Letters, 16, 2011 (2016).

21. K. Ushirogata, K. Sodeyama, Z. Futera, Y. Tateyama, and Y. Okuno, Journal of The Electrochemical Society, 162, A2670 (2015).

22. A. J. Smith, J. C. Burns, X. Zhao, D. Xiong, and J. R. Dahn, Journal of The Electrochemical Society, 158, A447 (2011).

23. P. Keil, S. F. Schuster, J. Wilhelm, J. Travi, A. Hauser, R. C. Karl, and A. Jossen, Journal of The Electrochemical Society, 163, A1872 (2016).
24. P Verma, P. Maire, and P. Novák, Electrochimica Acta, 55, 6332 (2010).

25. P. Ganesh, P. R. C. Kent, and D. E. Jiang, Journal of Physical Chemistry C, 116, 24476 (2012)

26. F. Single, B. Horstmann, and A. Latz, Phys. Chem. Chem. Phys., 18, 17810 (2016).

27. J. Christensen and J. Newman, Journal of The Electrochemical Society, 151, A1977 (2004).

28. D. Li, D. Danilov, Z. Zhang, H. Chen, Y. Yang, and P. H. L. Notten, Journal of the Electrochemical Society, 162, A858 (2015).

29. Y. X. Lin, Z. Liu, K. Leung, L. Q. Chen, P. Lu, and Y. Qi, Journal of Power Sources, 309, 221 (2016).

30. M. B. Pinson and M. Z. Bazant, Journal of the Electrochemical Society, 160, A243 (2012).

31. H. J. Ploehn, P. Ramadass, and R. E. White, Journal of The Electrochemical Society, 151, A456 (2004)

32. M. Tang, S. Lu, and J. Newman, Journal of The Electrochemical Society, 159, A1775 (2012).

33. J. Newman and K. Thomas-Alyea, Electrochemical Systems, Electrochemical Society series (John Wiley \& Sons, 2004).

34. B. Horstmann, T. Danner, and W. G. Bessler, Energy \& Environmental Science, 6 1299 (2013).

35. D. Bothe and W. Dreyer, Acta Mechanica, 226, 1757 (2015)

36. S. de Groot and P. Mazur, Non-equilibrium Thermodynamics, Dover Books on Physics (Dover Publications, 1962).

37. A. Latz and J. Zausch, Electrochimica Acta, 110, 358 (2013).

38. M. Z. Bazant, Accounts of Chemical Research, 46, 1144 (2013).

39. J. Bockris, A. Reddy, and M. Gamboa-Aldeco, Modern Electrochemistry 2A: Fundamentals of Electrodics, Modern electrochemistry (Springer US, 2001).

40. M. Tang and J. Newman, Journal of The Electrochemical Society, 159, A281 (2012)

41. K. Leung, F. A. Soto, K. Hankins, P. B. Balbuena, and K. L. Harrison, The Journal of Physical Chemistry C, 120, 6302 (2016).

42. E. Peled, D. Bar Tow, A. Merson, A. Gladkich, L. Burstein, and D. Golodnitsky, Journal of Power Sources, 97-98, 52 (2001).

43. M. Safari and C. Delacourt, Journal of The Electrochemical Society, 158, 562 (2011).

44. R. Naejus, D. Lemordant, R. Coudert, and P. Willmann, The Journal of Chemical Thermodynamics, 29, 1503 (1997).

45. O. Borodin, G. D. Smith, and P. Fan, Journal of Physical Chemistry B, 110, 22773 (2006).

46. K. Hayamizu, Journal of Chemical and Engineering Data, 57, 2012 (2012).

47. M. Broussely, S. Herreyre, P. Biensan, P. Kasztejna, K. Nechev, and R. J. Staniewicz, Journal of Power Sources, 97-98, 13 (2001) .

48. P. Liu, J. Wang, J. Hicks-Garner, E. Sherman, S. Soukiazian, M. Verbrugge, H. Tataria, J. Musser, and P. Finamore, Journal of The Electrochemical Society, 157, A499 (2010).

49. R. C. Chaney, E. E. Lafon, and C. C. Lin, Phys. Rev. B, 4, 2734 (1971) 